\title{
Article \\ Improved lipophilicity and aqueous solubility prediction with composite graph neural networks
}

\author{
Oliver Wieder $^{1, *(\mathbb{D}}$, Mélaine Kuenemann ${ }^{2} \mathbb{D}$, Marcus Wieder $^{1}\left(\mathbb{D}\right.$, Thomas Seidel ${ }^{1} \mathbb{D}$, Christophe Meyer $^{2} \mathbb{D}$ and \\ Thierry Langer ${ }^{1}(\mathbb{D}$ \\ 1 University of Vienna, Department of Pharmaceutical Chemistry, Althanstraße 14, A-1090 Vienna, Austria \\ 2 Servier Research Institute - CentEx Biotechnology,125 Chemin de Ronde, 78290 Croissy-sur-Seine, France \\ * Correspondence: oliver.wieder@univie.ac.at
}

check for updates

Citation: Lastname, F.; Lastname, F.; Lastname, F. Improved lipophilicity and aqueous solubility prediction using composite graph neural networks. Preprints 2021, 1, 0. https://doi.org/

Received:

Accepted:

Published:

Publisher's Note: MDPI stays neutral with regard to jurisdictional claims in published maps and institutional affiliations.

\begin{abstract}
The accurate prediction of molecular properties such as lipophilicity and aqueous solubility is of great importance in several stages of the drug discovery pipeline. Machine learning methods like graph-based neural networks have shown exceptionally good performance in predicting these properties. In this work we introduce a novel graph neural network architecture composed of two distinct sub-architectures that achieves an improvement in accuracy over its individual parts employing various learning-, and featurization strategies. We argue that combining models with different key aspects might help make graph neural networks deeper while simultaneously increasing their predictive power. Additionally, we want to highlight the need to move beyond comparing single performance metrics to show machine learning model superiority.
\end{abstract}

Keywords: AI, deep-learning, neural-networks, graph neural-networks, cheminformatics, molecular property, machine-learning, computational chemistry, lipophilicity, solubility

\section{Introduction}

Oral bio-availability, drug uptake and ADME-related properties of small molecules are key properties in pharmacokinetics. For drugs to reach their intended target, they need to pass through several barriers either by passive diffusion or carrier-mediated uptake typically mediated by lipophilicity and aqueous solubility. Drugs with poor solubility are unable to achieve that and therefore pose a higher risk in attrition and overall cost during their development [1]. Methods based on deep-learning have proven successful in predicting molecular properties [2] and are becoming more and more part of the modern computer-aided drug design toolbox. Since molecules can be represented as graphs, an obvious approach is to employ a graph-based architecture for deep-learning - which leads to the utilization of graph based neural networks (GNNs). These kinds of networks are capable of learning representations for a specific task in an automated way and can therefore eliminate the complicated feature engineering process where domain specialists have to select the list of descriptors themselves [3]. They gained more and more attention and became increasingly popular in the last few years [4][5][6] especially due to their success in outperforming traditional machine learning methods for certain tasks [7-12]. One of the first GNN models used for physicochemical property prediction was Micheli [13] in 2009. They predicted the boiling point of alkanes with a recursive architecture for structured data input and achieved improved state-of-the-art performance. Lusci et al. [14] were the first to apply an undirected cyclic graph recurrent neural network successfully on predicting aqueous solubility. In the following years several recurrent, spatial and spectral graph based neural networks were introduced [15], [16], [17] and [3]. Surveys like [18] introduced easy to use benchmark data sets for physicochemical properties using different neural network architectures. Another successful GNN framework is the message passing framework introduced by [3] and then extended by [1] to include directed edges. These networks were successful in predicting several chemical properties including lipophilicity and aqueous solubility. Nevertheless, one remaining problem with vanilla GNNs is that they are unaware of the structural role of each node or edge [19], called the graph isomorphism problem. Most standard GNNs such as MPNN are incapable of 
distinguishing between different types of graph structures to determine whether they are topologically identical [20]. To address this issue, a group of GNNs have recently received additional attention namely graph isomorphism networks (GIN) [20]. These networks try to solve the isomorphism problem for graphs by reformulating the message passing framework to incorporate the Weisfeiler-Lehman (WL) hierarchy. They try to be at least as expressive as the Weisfeiler-Lehman graph isomorphism test (WL-test) [21] and have shown exceptionally good results [20],[22] despite often being computationally expensive [23]. Inspired by the success of the GIN and the directed message passing neural network (D-MPNN) framework we combined the key aspects of both architectures to perform lipophilicity and aqueous solubility predictions. Additionally, when comparing new machine learning architectures with previously published methods, the standard approach is to compare single performance metrics like root mean squared error (RMSE) values with each other in order to show model superiority [24][23]. This can often be problematic as stochastic algorithms like neural networks can vary greatly in their prediction even without changing their hyperparameters simply by using different training/validation/test set splits or non deterministic weight initialization [25][26]. One of the reasons for this is the complex landscape that optimizers have to navigate through in modern machine learning models. In real world applications these landscapes can have multiple local minimas and it is especially hard for non-deterministic optimization algorithms like stochastic gradient descent to find the global minimum, therefore often retrieving different results when repeated [27]. his problem can be intensified by using small data sets with different random splits for training and evaluation. Such an approach can lead the optimization algorithm into different local minimums and makes it almost impossible for the model to generalize [2]. It is therefore difficult to compare different model architectures with each other even when using the same data [25]. Another challenge is especially prominent in the GNN domain, where the optimal features for node or edge representation are unknown. Surveys often use the same data but different representations for their input data which makes it difficult to make a fair comparison between the models [2][3] . In order to mitigate these problems we use the same data points for each of the algorithms to train, evaluate and test but also retrain each of the used models with different node and edge features as well as learning strategies to obtain an average performance independent of the used features and training approaches. This approach is time consuming as multiple models have to be evaluated several times. Nevertheless, obtaining a better understanding of the optimale parameters defining the GNNs will facilitate the understanding of these architectures and ultimately help move GNNs beyond the current hype to more explainable and robust models. Our contribution is a novel graph neural network architecture called directed-edge graph isomorphism network (D-GIN). It extends the directed-edge message passing (D-MPNN) framework described in [1] by the graph isomorphism network (GIN) published in [20]. An overview of the model is shown in figure 1. Our novel architecture shows improved performance compared to its individual, less complex networks and we argue that combining models with different key aspects might help make graph neural networks deeper while simultaneously increasing their predictive power. 
Figure 1. High level representation of the D-GIN architecture. Panel (a) shows the high level workflow. A graph and its nodes and edges is featurized, then fed into the directed-edge graph isomorphism network (D-GIN) to generate a molecular embedding for the entire graph. This novel representation is then used for the physical property prediction. Panel (b) shows the DGIN architecture at a low level. First the hidden directed-edge features $\left(h_{u v}^{0}\right)$ are initialized by concatenating the corresponding node $\left(x_{v}\right)$ and directed-edge $\left(x_{u v}\right)$ features. Then directed-edge messages $\left(m_{u v}\right)$ are used to update the hidden directed-edge features $\left(h_{u v}^{t}\right)$. The directed messages are then combined with their corresponding hidden node features $\left(h_{v}\right)$ and iteratively updated by an additional trainable identifier called epsilon. It is used to differentiate between similar nodes. The hidden node features are then aggregated to generate the molecular embedding $\left(h_{G}\right)$ which is used as input for the feed-forward neural network to predict the physico-chemical properties $(\log \mathrm{D}, \log S$ or $\log \mathrm{P})$.

\section{(a)}

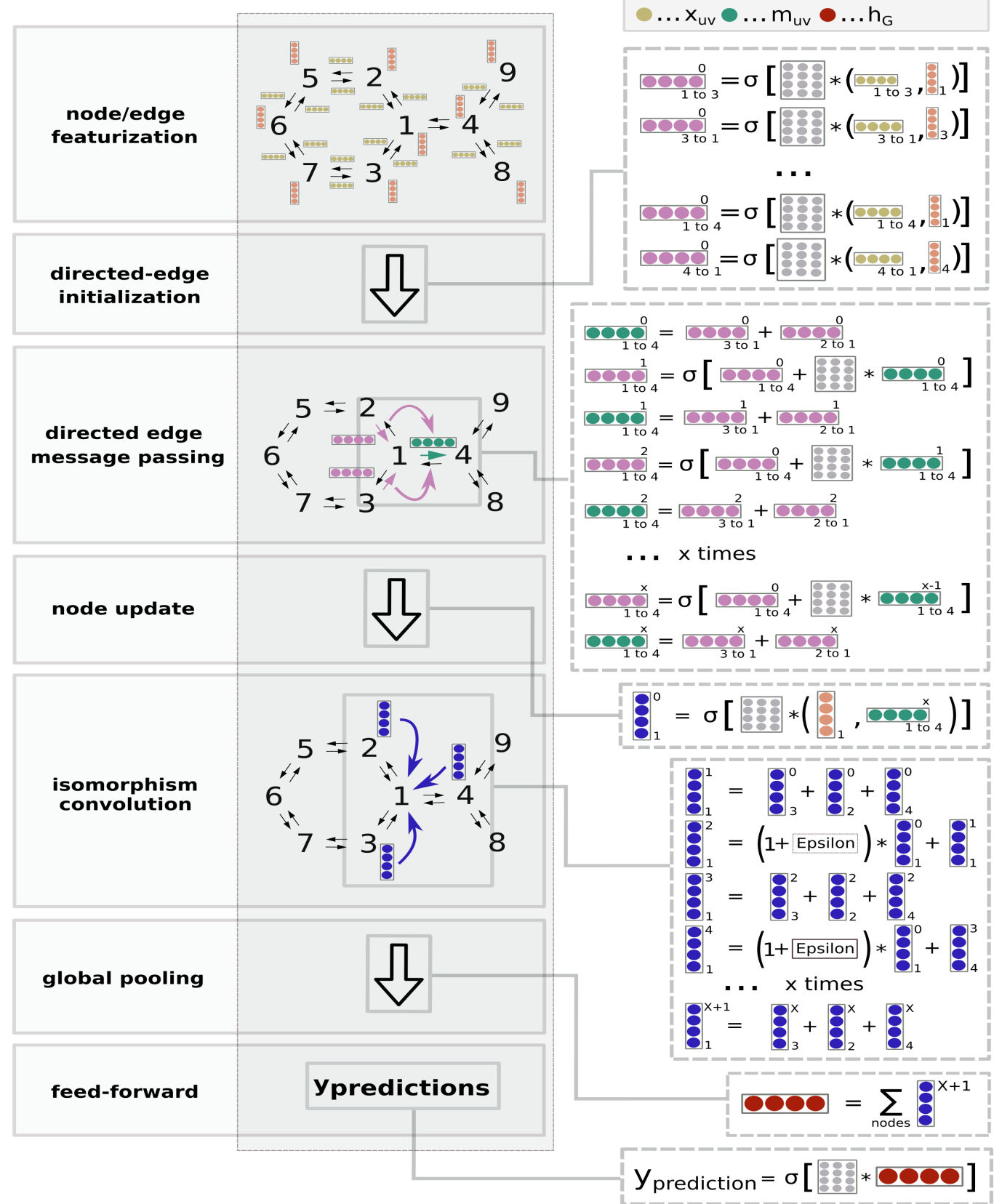

(b) $\bullet \ldots x_{v} \bullet \ldots h_{u v} \bullet \ldots h_{v} \quad \ldots w$

$$
\ldots x_{\mathrm{uv}} \bigcirc \ldots \mathrm{m}_{\mathrm{uv}} \odot \ldots \mathrm{h}_{\mathrm{G}}
$$

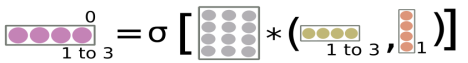

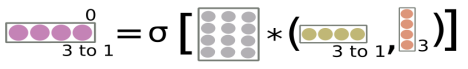

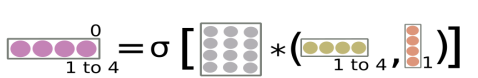

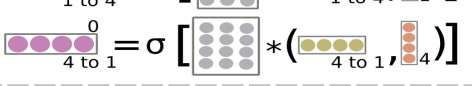

$\frac{0000^{0}}{1 \text { to } 4}=\frac{0000}{3 \text { to } 1}+\frac{0000}{2 \text { to } 1}$

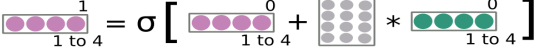
${ }_{1 \text { to } 4}=\frac{0000^{1}}{3 \text { to } 1}+\frac{0000}{2 \text { to } 1}$ 1 to $4=\sigma\left[\begin{array}{ll}1 \text { to } 4 & +88\end{array}\right.$ 3 to $1 \quad 2$ to

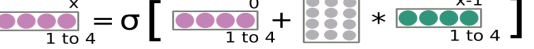
3 to 1 


\section{Materials and methods}

This section gives a detailed overview over the used data, molecular representation and the different machine learning methods used throughout this work. The most common notations are shown in table 1.

Table 1: Common notations used throughout this publication.

\begin{tabular}{ll} 
Notations & Definitions \\
\hline$\tau$ & A non-linear function (e.g. sigmoid or relu) \\
cat $()$, & Vector concatenation \\
$t$ & Iterator of t steps \\
$G$ & A graph \\
$V$ & Set of nodes \\
$E$ & Set of edges \\
$v$ & Node $v \in V$ \\
$e_{u v}$ & Edge $e_{u v} \in E$ between node $u$ and $v$ \\
$N(u)$ & Neighbors of node $u$ \\
$N(u) / w$ & Neighbors of node $u$ except $w$ \\
$n$ & The number of nodes \\
$m$ & The number of edges \\
$d$ & The dimension of a node feature vector \\
$b$ & The dimension of a edge feature vector \\
$X \in^{n \times d}$ & Feature matrix of a graph \\
$x_{v} \in^{d}$ & Feature vector of node $v$ \\
$x^{e} u v$ & Feature vector of edge $e_{u v}$ \\
$h_{v} \in \in^{c}$ & Hidden feature vector of node $v$ \\
$m_{v} \in^{c}$ & Message feature vector to node $v$ \\
$h_{G} \in \in^{c}$ & Feature vector of the graph $G$ \\
$h_{u v} \in^{d}$ & Hidden feature vector of edge $e_{u v}$ \\
$m_{u v} \in^{d}$ & Message feature vector to edge $e_{u v}$ \\
$W$ & Weight matrix of a neural network \\
$A \in\{1,0\}$ & Adjacency matrix \\
$R M S E$ & Root mean squared error \\
$G N N$ & Graph neural network \\
$G I N$ & Graph isomorphic network as in [20] \\
$\epsilon$ & Epsilon as described in [20] \\
$D-M P N N$ & directed-edge message passing network as in [1] \\
$D-G I N$ & directed-edge graph isomorphic network \\
$C I$ & 95\% confidence interval calculated via bootstrapping \\
$f(\cdot)$ & Feed forward neural network \\
&
\end{tabular}

\subsection{Experimental data}

Two data sets were used to predict three different properties $(\log S, \log \mathrm{D}$ and $\log \mathrm{P}$ values). The first data set is the Delaney lipophilicity data set [18] containing experimentally evaluated $\log \mathrm{D}$ and $\log \mathrm{P}$ values at $\mathrm{pH}$ 7.4. The second set is an aqueous solubility data set consisting of experimentally evaluated logS in combination with their corresponding $\log \mathrm{P}$ value and was described in [28]. We split each of the data sets randomly into three subsets for training, evaluation and testing. This was done in a ratio of 81:9:10 for the training/evaluation and test set. Molecules in both sets were neutralized and the preprocessed data can be found in the GitHub repository[29]. The lipophilicity data set consists of 4174 compounds, 3380 were used for training, 376 for evaluating and model selection and 418 for testing. The aqueous solubility data set was cleaned, stripped off compounds with more than one molecule (e.g. salts) and those lower than -10.0 and higher than $0.0 \log S$ units. Finally 6444 molecules were used in this work. We applied the same training, evaluation and test split as for the lipophilicity data set and got 5219 compounds for training, 579 for 
evaluating and model selection and 645 for testing. The data split was performed with the same seed for each of the models, training and featurization strategies to be able to compare the different models with the exact same training, evaluation and test data. The minimum value of each of the $\log \mathrm{D}, \log \mathrm{P}$ and $\log \mathrm{S}$ properties was used as an offset to ensure only positive property values.

\subsection{Training approaches}

The training strategies differ in the used data set and the training target $(\log \mathrm{D}, \log \mathrm{P}$ or $\log S$ ). Under these constraints seven different types of strategies are being used. The first multi-task learning strategy uses a combined approach of $\log \mathrm{D}, \log \mathrm{P}$ and $\log \mathrm{S}$ values during training - this approach is commonly referred to as " $\log \mathrm{D} / \mathrm{S} / \mathrm{P}$ ". Three additional multi-task strategies utilizes a combination of two physico-chemical properties and are referred to as either " $\log \mathrm{D} / \mathrm{P}$ ", " $\log \mathrm{D} / \mathrm{S}$ " or " $\log \mathrm{S} / \mathrm{P}$ " depending on the used endpoints. Additional three single task strategies only learn on a single physico-chemical property and are referred to as either "single task $\log \mathrm{D}^{\prime}$, , $\log \mathrm{P}^{\prime}$ or " $\log \mathrm{S}^{\prime}$. When physico-chemical properties from different data sets are used, the individual data sets are first split into training, evaluation and test sets. Afterwards each physico-chemical property is evaluated and tested individually so that the evaluation and test results of the multi-task learning approaches can be compared to those with a single-task learning strategy. When testing either single-, or multi-task models not the combined but the individual RMSE was taken as the measure for the best model. For $\log \mathrm{P}$ we only used the results from either the first multi-task approach ("multitask $\log \mathrm{S} / \mathrm{D} / \mathrm{P}$ ") or the single-task approach with $\log \mathrm{P}$ values. The reasoning behind this was to use the exact same test and evaluation data for all models while trying to avoid an unbalanced data bias in favour of $\log \mathrm{P}$ values. When training with two physico-chemical properties where one was $\log \mathrm{P}$, we only used the data that had both properties.

\subsection{Molecular graphs}

A graph is defined as $G=(V, E)$, where $V$ is a set of nodes and $E$ denotes a set of edges. Let $v \in V$ be a node with feature vector $x_{v}$ and $e_{u v} \in E$ be an edge pointing from $u$ to $v$ with feature vector $x_{u v}^{e}$. The adjacency matrix $A$ shows the connectivity of the nodes and in our case it is binary as we do not weigh any connections. It is defined as a $n \times n$ matrix with $A_{u v}=1$ if $e_{u v} \in E$ and $A_{u v}=0$ if $e_{u v} \notin E$. We use directed, heterogeneous graphs where $e_{u v} \neq e_{v u}$. Heterogeneous graphs contain different types of nodes and edges with their corresponding featurizations.

\subsection{Molecular featurization}

Five different types of edge and vertex featurizations $X$ are being used for the GNNs. The detailed description of $x$ and $x^{e}$ can be found in tables 2 to 7 . The feature vectors for the non-GNN models consist of 8 different settings - fingerprints (ECFP or MACCSKeys - shown in table 13 in the SI) used either in combination with standardized RDKit[30] descriptors or without the descriptors. The descriptors were a combination of all possible and standardized RDKit descriptors, which had a total length of 208. The parametrization of the ECFP was either 1024, 1536 or 2048 bits with a radius of 4 . Featurization 3 (table 2) and 4 (table 3 ) only differ in the way the size of ring systems are being represented. Either as a float value calculated by 1 divided by the size of the ring or as a one-hot encoding with 10 possibilities shown in 3 . Both representations apply the maximum featurization used in this work of both edges and nodes. The node and edge featurization in 5 (table 4) include two node features (atom type and formal charge) and one edge feature (bond order). Featurization 6 (table 5) includes the same node description as 5 and the edge featurization of 3. Featurization 7 (table 6) has the same node featurization as 3 and the same edge featurization as 5 . Featurization 8 (table 7 ) includes a set of optimized node and edge features. This was done by using a trained D-GIN model and then removing one node or edge feature at a time and observing the RMSE of the prediction. The five 
node features and the three edge features that had the biggest impact on the RMSE were then taken as the featurization. The graphs and its featurizations were implemented using python version 3.7.8 and the toolkit CDPKit[31].

Table 2: Node and edge featurization of type 3.

\begin{tabular}{llc}
\hline Feature Name & Description & Size \\
\hline \hline atom type & type of the atom (H,C,N,O,S,F,P,CL,BR,I) & 10 \\
formal charge & calculated formal charge $(-2,-1,0,1,2)$ & 5 \\
CIP & configuration of each atom (R,S,None,either) & 4 \\
Hybridization & atom's hybrdiization state (sp,sp2,sp3,sp3d,sp3d2,none) & 6 \\
amide center & is the atom the center of an amide & 2 \\
aromaticity & is the atom in an aromatic & 2 \\
ring size & if in ring, what size (float 1/size) & 1 \\
nr. of hydrogens & get the number of implicit hydrogens (0-5) & 6 \\
\hline bond order & order of the bond (1,2,3) & 3 \\
conjugated & is the bond conjugated & 2 \\
rotable & is the bond rotable & 2 \\
amide bond & is the bond in an amide & 2 \\
aromaticity & is the bond in an aromatic & 2 \\
ring flag & is the bond in a ring & 1 \\
ring size & if in ring, what size (float 1/size) & 6 \\
CIP & configuration of each bond (None,E,Z,Trans,Cis,either) & 2 \\
\hline \hline
\end{tabular}

Table 3: Node and edge featurization of type 4.

\begin{tabular}{llc}
\hline Feature Name & Description & Size \\
\hline \hline atom type & type of the atom $(\mathrm{H}, \mathrm{C}, \mathrm{N}, \mathrm{O}, \mathrm{S}, \mathrm{F}, \mathrm{P}, \mathrm{CL}, \mathrm{BR}, \mathrm{I})$ & 10 \\
formal charge & calculated formal charge $(-2,-1,0,1,2)$ & 5 \\
CIP & configuration of each atom $(\mathrm{R}, \mathrm{S}, \mathrm{None}, \mathrm{either})$ & 4 \\
Hybridization & atom's hybrdiization state $(\mathrm{sp}, \mathrm{sp} 2, \mathrm{sp} 3, \mathrm{sp} 3 \mathrm{~d}, \mathrm{sp} 3 \mathrm{~d} 2, \mathrm{none})$ & 6 \\
amide center & is the atom the center of an amide & 2 \\
aromaticity & is the atom in an aromatic & 2 \\
ring size & if in ring, what size $(0,3,4,5,6,7,8,9,10,11)$ & 10 \\
nr. of hydrogens & get the number of implicit hydrogens $(0-5)$ & 6 \\
\hline bond order & order of the bond (1,2,3) & 3 \\
conjugated & is the bond conjugated & 2 \\
rotable & is the bond rotable & 2 \\
amide bond & is the bond in an amide & 2 \\
aromaticity & is the bond in an aromatic & 2 \\
ring flag & is the bond in a ring & 10 \\
ring size & if in ring, what size $(0,3,4,5,6,7,8,9,10,11)$ & 6 \\
CIP & configuration of each bond (None,E,Z,Trans,Cis,either) \\
\hline \hline
\end{tabular}


Table 4: Node and edge featurization of type 5.

\begin{tabular}{llc}
\hline Feature Name & Description & Size \\
\hline \hline atom type & type of the atom $(\mathrm{H}, \mathrm{C}, \mathrm{N}, \mathrm{O}, \mathrm{S}, \mathrm{F}, \mathrm{P}, \mathrm{CL}, \mathrm{BR}, \mathrm{I})$ & 10 \\
formal charge & calculated formal charge $(-2,-1,0,1,2)$ & 5 \\
$\mathrm{CIP}$ & - & - \\
Hybridization & - & - \\
amide center & - & - \\
aromaticity & - & - \\
ring size & - & - \\
nr. of hydrogens & - & - \\
\hline bond order & order of the bond $(1,2,3)$ & 3 \\
conjugated & - & - \\
rotable & - & - \\
amide bond & - & - \\
aromaticity & - & - \\
ring flag & - & - \\
ring size & - & - \\
CIP & - & - \\
\hline \hline
\end{tabular}

Table 5: Node and edge featurization of type 6.

\begin{tabular}{llc}
\hline Feature Name & Description & Size \\
\hline \hline atom type & type of the atom (H,C,N,O,S,F,P,CL,BR,I) & 10 \\
formal charge & calculated formal charge $(-2,-1,0,1,2)$ & 5 \\
CIP & - & - \\
Hybridization & - & - \\
amide center & - & - \\
aromaticity & - & - \\
ring size & - & - \\
nr. of hydrogens & - & - \\
\hline bond order & order of the bond (1,2,3) & 3 \\
conjugated & is the bond conjugated & 2 \\
rotable & is the bond rotable & 2 \\
amide bond & is the bond in an amide & 2 \\
aromaticity & is the bond in an aromatic & 2 \\
ring flag & is the bond in a ring & 2 \\
ring size & if in ring, what size (float 1/size) & 1 \\
CIP & configuration of each bond (None,E,Z,Trans,Cis,either) & 6 \\
\hline \hline
\end{tabular}


Table 6: Node and edge featurization of type 7.

\begin{tabular}{llc}
\hline Feature Name & Description & Size \\
\hline \hline atom type & type of the atom (H,C,N,O,S,F,P,CL,BR,I) & 10 \\
formal charge & calculated formal charge (-2,-1,0,1,2) & 5 \\
CIP & configuration of each atom (R,S,None,either) & 4 \\
Hybridization & atom's hybrdiization state (sp,sp2,sp3,sp3d,sp3d2,none) & 6 \\
amide center & is the atom the center of an amide & 2 \\
aromaticity & is the atom in an aromatic & 2 \\
ring size & if in ring, what size (float 1/size) & 1 \\
nr. of hydrogens & get the number of implicit hydrogens (0-5) & 6 \\
\hline bond order & order of the bond (1,2,3) & 3 \\
conjugated & - & - \\
rotable & - & - \\
amide bond & - & - \\
aromaticity & - & - \\
ring flag & - & - \\
ring size & - & - \\
CIP & - &
\end{tabular}

Table 7: Node and edge featurization of type 8.

\begin{tabular}{llc}
\hline Feature Name & Description & Size \\
\hline \hline atom type & type of the atom (H,C,N,O,S,F,P,CL,BR,I) & 10 \\
formal charge & calculated formal charge $(-2,-1,0,1,2)$ & 5 \\
CIP & configuration of each atom (R,S,None,either) & 4 \\
Hybridization & atom's hybrdiization state (sp,sp2,sp3,sp3d,sp3d2,none) & 6 \\
amide center & - & - \\
aromaticity & - & - \\
ring size & if in ring, what size (float 1/size) & 1 \\
nr. of hydrogens & - & - \\
\hline bond order & order of the bond $(1,2,3)$ & 3 \\
conjugated & - & - \\
rotable & is the bond rotable & 2 \\
amide bond & - & - \\
aromaticity & - & 2 \\
ring flag & is the bond in a ring & - \\
ring size & - & - \\
CIP & - & \\
\hline \hline
\end{tabular}




\subsection{Directed-edge GIN (D-GIN) and reference models}

The D-GIN is an extension of the message passing neural network of [1] without the additional feature engineering in combination with the graph isomorphic network (GIN) of [20]. Its high level representation can be seen in figure 1 Altogether the DGIN is initialized as

$$
h_{u w}^{0}=\tau\left(W_{\text {init }}\left(\operatorname{cat}\left(x_{u}, x^{e}{ }_{u w}\right)\right)\right)
$$

followed by a $t \in 1, \ldots, T$ iteration of

$$
\begin{gathered}
m_{u w}^{(t+1)}= \begin{cases}\sum_{k \in N(u) / w} h_{k u^{\prime}}^{0} & \text { if } t==0 . \\
\sum_{k \in N(u) / w} h_{k u^{\prime}}^{t} & \text { otherwise. }\end{cases} \\
h_{u w}^{(t+1)}=\tau\left(h_{u w}^{0}+W_{m} m_{u w}^{t+1}\right)
\end{gathered}
$$

after which the messages for each directed-edge is being summed as

$$
m_{u}=\sum_{w \in N(u)} h_{u w}^{T}
$$

then the message $m_{u}$ is being concatenated as

$$
h_{u}= \begin{cases}\operatorname{cat}\left(m_{u}, x_{u}\right), & \text { if D-GIN. } \\ \left(W_{a g g}\left(\operatorname{cat}\left(m_{u}, x_{u}\right)\right),\right. & \text { if D-MPNN. }\end{cases}
$$

and another message passing over $l \in 1, \ldots, T_{2}$ is performed by

$$
\begin{gathered}
h_{u}^{(l)}= \begin{cases}\sum_{w \in N(u)} h_{w}, & \text { if D-GIN. } \\
x_{u}, & \text { if GIN. }\end{cases} \\
h_{u}^{(l+1)}=\left(W_{a g g}(1+\epsilon) h_{u}^{0}+h_{u}^{(l)}\right)
\end{gathered}
$$

afterwards the updated feature vectors $h_{\text {node }}^{T}$ of each node are being aggregated over the whole molecule as

$$
h_{G}=\sum_{h \in\left(H^{(T)}\right)} h
$$

The readout phase is then defined as $\hat{y}=f\left(h_{G}\right)$ where $f(\cdot)$ is a feed-forward neural network. The D-MPNN consists of Equations 1 to 5 but then uses the hidden feature vectors for each node directly by applying Equation (5) and then immediately Equation (7) to encode the whole graph as $h_{G}$. The GIN on the other hand is initialized and trained as shown in Equation (6) (if GIN) in order to update the hidden feature vectors of each node. Afterwards 1 update steps, the hidden feature vector of each node serves as the input of Equation (7) to get the aggregated representation $h_{G}$ for the whole graph. The D-GIN uses all of these functions in a combined way described above (Equations 1 to 8).

\subsection{Graph neural network implementation, training and hyper-parameter search}

All GNNs have been implemented and trained using tensorflow 2.3.0 [32]. We conducted a hyper-parameter-search to find the best parameters which were further used to train all models. Further details on the hyper-parameters are given in the corresponding model's configuration files accesable via [29] in the .resultsconfigsmodel_name folders. Each GNN model type was trained twice with 42 different settings depending on the training strategy and featurization - in total 84 training runs per model type were performed. Each non-GNN model type was trained with 8 different settings. For training, evaluation 
and testing we split each of the data sets as described in section Experimental data. Each of the GNNs were trained for 1600 epochs and the model with the best performance on the validation set was saved (RMSE was used as an evaluation metric). Additionally we calculated r-squared values. To evaluate the model type performance we used the model with the best RMSE out of the two runs performed for each model setting. When evaluating the average model type performance, the average RMSE over all 42 different model settings was used for the calculation. In order to evaluate models with several properties, we summed all RMSEs and when the combined RMSE on the evaluation set was below the last best RMSE, the model weights were safed. We used these models to test the model on the test set. Each model was run two times and the results with the best test set performance were taken. Additionally, the 95\% confidence interval range was calculated by applying bootstrapping 100 times while leaving out $10 \%$ of the test data set. We also combined the best GNN model for each physical property with the best non-GNN model. We did this by adding the predicted log values of one model with the other and then divided it by two. These hybrid models are then called according to their GNN model type plus consensus (e.g. D-GIN cons.).

\subsection{Other machine learning approaches}

We used the random forest (RF), support vector machine (SVM) and k-nearest neighbour (K-NN) implementations of scikit-learn (Version 0.23.2 [33]). Default hyperparameters were used. The featurization is described in table 13. When using descriptors as input, we standardized them with the scikit-learn StandardScaler - it removes the mean and scales to unit variance. For the fingerprints and descriptors we used version 2020.09.2 of the RDKit [30] python package. Each of the models have been trained in a single-task manner for each of the log values. 


\section{Results and discussion}

In the following we briefly describe certain terms used throughout this publication that might have ambiguous meaning to prevent misunderstandings. The term "model type" is used to refer to different kinds of machine learning algorithms, e.g. a model type can be a RF, SVM, KNN, D-GIN, GIN or D-MPNN. We further refer to a trained model instance with particular training and featurization strategies simply as "model". The term "training strategy" is used to distinguish between different single- and multi-task training approaches trained with a combination of the molecular properties - e.g. $\log \mathrm{D} / \mathrm{S} / \mathrm{P}$ is used to show that $\log \mathrm{D}, \log \mathrm{S}$ and $\log \mathrm{P}$ were used during training. They are described in section Training approaches. The term "featurization strategy" is used to describe the different node and edge features used for the models to train on. They are described in table tables 2 to 7 . We additionally distinguish between consensus and non-consensus models. These hybrid models are a combination of the best GNN and the best non-GNN models - SVM D-GIN for $\log D$ and $\log S$ and RF D-GIN for $\log$ P. To obtain consensus predictions, values of the two models were averaged. These values are then used as "new" predictions for the RMSE calculation and are called according to their GNN model type plos cons. - e.g. D-GIN cons.

The results and discussion section is split into two parts: The first part (General model performance) compares different GNNs and non-GNN methods used in this work to identify the best overall performing model type. In the second part (Impact of molecular featurization and training strategies), we will investigate the impact of different learning strategies (i.e. Multi-task vs single task learning) as well as different featurizations on the performance. Overall 6 different machine learning model types were used in this survey. The three GNN model types are D-MPNN, GIN and D-GIN. The three non-GNN model types are random forest (RF) regression, support vector machines (SVM) and k-nearestneighbor (KNN) algorithm. Each model type was trained with the same hyperparameters but 7 different learning strategies and 6 different node/edge featurization strategies to predict $\log \mathrm{D}, \log \mathrm{P}$ and $\log \mathrm{S}$ values. We trained each GNN model type with each physical property with all 42 strategies two times and used the models with the best evaluation RMSE for testing. Overall 84 training runs were performed for each of the GNN model types. The non-GNN models had 8 different featurization strategies for each model type. The training set for the three different physical properties contained $4174 \log \mathrm{D}, 6444 \log S$ and $10618 \log \mathrm{P}$ experimentally measured values. The same training/evaluation/test set was used for all GNN and non-GNN model types.

\subsection{General model performance}

In this section we present the results for the different model types for each of the property predictions. Table 8 gives an overview over the best models with RMSE and $r^{2}$ results for the $\log \mathrm{D}, \log \mathrm{P}$ and $\log \mathrm{S}$ predictions for each model type. Additionally it shows what kind of training strategy and featurization was used. The dark gray areas highlight the best model for the particular property, the light gray area shows the second best model and the red asterisk marks the best non-consensus model. Overall, the consensus models perform best for each of the physical property predictions. Among the non-consensus models, the D-GIN model type outperforms the other GNN architectures for each property prediction but not the non-GNN architectures. For $\log P$ and $\operatorname{logS}$ prediction SVM and RF models outperform the GNN architecture. 
Table 8: The best performing model types with corresponding training strategy, featurization, log RMSE and $r^{2}$ values are reported on the hold out test set. The models with dark gray background show the best performance for the property prediction whereas the light gray represent the second best models. The red asterisks mark the lowest RMSE for the non-consensus models for each property prediction. For each property three sections of rows are shown: top section for the different GNN architectures (D-GIN, D-MPNN and GIN), middle section for the SVM, RF and KNN model types and the bottom section for the consensus models combining the best GNN model and non-GNN models.

\begin{tabular}{|c|c|c|c|c|c|}
\hline $\begin{array}{l}\text { molecular } \\
\text { property }\end{array}$ & model type & $\begin{array}{l}\text { training } \\
\text { strategy }\end{array}$ & $\begin{array}{l}\text { featurization } \\
\text { strategy }\end{array}$ & RMSE & $r^{2}$ \\
\hline \multirow{9}{*}{$\log \mathrm{D}$} & D-GIN & $\log \mathrm{D} / \mathrm{P}$ & 6 & $0.553 \pm 0.049^{*}$ & $0.766 \pm 0.053$ \\
\hline & D-MPNN & $\log \mathrm{D} / \mathrm{P}$ & 6 & $0.686 \pm 0.054$ & $0.645 \pm 0.071$ \\
\hline & GIN & $\log \mathrm{D}$ & 8 & $0.734 \pm 0.058$ & $0.589 \pm 0.079$ \\
\hline & SVM & $\log \mathrm{D}$ & 17 & $0.639 \pm 0.051$ & $0.685 \pm 0.046$ \\
\hline & RF & $\log \mathrm{D}$ & 15 & $0.699 \pm 0.062$ & $0.623 \pm 0.058$ \\
\hline & KNN & $\log \mathrm{D}$ & 17 & $0.801 \pm 0.060$ & $0.506 \pm 0.058$ \\
\hline & D-GIN cons. & $\log \mathrm{D} / \mathrm{P}$ & $6 \& 17$ & $0.548 \pm 0.051$ & $0.768 \pm 0.049$ \\
\hline & D-MPNN cons. & $\log \mathrm{D} / \mathrm{P}$ & $6 \& 17$ & $0.613 \pm 0.055$ & $0.710 \pm 0.057$ \\
\hline & GIN cons. & $\log \mathrm{D}$ & $8 \& 17$ & $0.627 \pm 0.054$ & $0.698 \pm 0.059$ \\
\hline \multirow{9}{*}{$\log S$} & D-GIN & All & 8 & $0.795 \pm 0.037$ & $0.807 \pm 0.018$ \\
\hline & D-MPNN & $\log \mathrm{D} / \mathrm{S}$ & 6 & $0.857 \pm 0.034$ & $0.781 \pm 0.015$ \\
\hline & GIN & $\log S / P$ & 7 & $1.088 \pm 0.065$ & $0.643 \pm 0.035$ \\
\hline & SVM & $\log S$ & 17 & $0.729 \pm 0.040^{*}$ & $0.837 \pm 0.020$ \\
\hline & RF & $\log S$ & 17 & $0.760 \pm 0.042$ & $0.823 \pm 0.020$ \\
\hline & KNN & $\log S$ & 14 & $1.057 \pm 0.055$ & $0.657 \pm 0.031$ \\
\hline & D-GIN cons. & All & $8 \& 17$ & $0.704 \pm 0.039$ & $0.847 \pm 0.013$ \\
\hline & D-MPNN cons. & $\log \mathrm{D} / \mathrm{S}$ & $6 \& 17$ & $0.743 \pm 0.037$ & $0.830 \pm 0.012$ \\
\hline & GIN cons. & $\log \mathrm{D} / \mathrm{P} / \mathrm{S}$ & $7 \& 17$ & $0.825 \pm 0.037$ & $0.791 \pm 0.015$ \\
\hline \multirow{9}{*}{$\log P$} & D-GIN & $\log \mathrm{P}$ & 3 & $0.472 \pm 0.019$ & $0.895 \pm 0.008$ \\
\hline & D-MPNN & $\log P$ & 6 & $0.540 \pm 0.019$ & $0.862 \pm 0.012$ \\
\hline & GIN & $\log \mathrm{D} / \mathrm{P} / \mathrm{S}$ & 4 & $0.716 \pm 0.032$ & $0.758 \pm 0.018$ \\
\hline & RF & $\log P$ & 16 & $0.470 \pm 0.023^{*}$ & $0.896 \pm 0.010$ \\
\hline & SVM & $\log \mathrm{P}$ & 17 & $0.493 \pm 0.029$ & $0.885 \pm 0.013$ \\
\hline & KNN & $\log \mathrm{P}$ & 17 & $0.743 \pm 0.019$ & $0.739 \pm 0.014$ \\
\hline & D-GIN cons. & $\log \mathrm{P}$ & $3 \& 16$ & $0.428 \pm 0.025$ & $0.913 \pm 0.009$ \\
\hline & D-MPNN cons. & $\log \mathrm{D} / \mathrm{P} / \mathrm{S}$ & $6 \& 16$ & $0.443 \pm 0.024$ & $0.907 \pm 0.009$ \\
\hline & GIN cons. & $\log \mathrm{P}$ & $4 \& 16$ & $0.533 \pm 0.039$ & $0.865 \pm 0.018$ \\
\hline
\end{tabular}

The best result for $\log \mathrm{D}$ is obtained by a D-GIN consensus model (D-GIN and SVM) with a RMSE of 0.5480 .051 followed by a D-GIN model with a RMSE of 0.553 0.049. The improvement achieved here with the consensus model is low compared to the other two properties. The best results for logS are also obtained by a D-GIN consensus model (D-GIN and SVM) with a RMSE of 0.7040 .039 followed by a SVM with a RMSE of 0.729 0.040. The best GNN model is a D-GIN with a RMSE of 0.7950 .037 . The improvement achieved by combining the two best models for the logS (D-GIN and SVM) is higher than for $\log \mathrm{D}$ but still lower than for the $\log \mathrm{P}$ results. For the latter property the best model is a D-GIN consensus model (D-GIN and RF) with a RMSE of 0.4280 .025 followed by another consensus model, the D-MPNN in combination with a RF model. The best GNN model is a D-GIN model with a RMSE of 0.4720 .019 , which performs similarly to the best non-GNN model (RF) with a RMSE of 0.4700 .023 .

Overall the best non-GNN methods perform well compared to the best GNN model types. This is true comparing the individual models with each other. When the average 
over all applied learning-, and featurization strategies are considered (see figures 2, 3, 4 and table 9), the non-GNN model types perform on average worse.

Table 9: The average performance of each model type for each property prediction independent of training and featurization strategy. It was calculated using all 42 different model settings. The dark and light gray boxes mark the best and second best model types for the physical property. Red asterisks mark the best average non-consensus models.

\begin{tabular}{|c|c|c|c|c|}
\hline $\begin{array}{l}\text { molecular } \\
\text { property }\end{array}$ & model type & $\begin{array}{l}\text { mean } \\
\text { RMSE }\end{array}$ & $\underset{\text { RMSE }}{\min }$ & $\begin{array}{c}\max \\
\text { RMSE }\end{array}$ \\
\hline \multirow{9}{*}{$\log \mathrm{D}$} & D-GIN & $0.615 \pm 0.039^{*}$ & 0.553 & 0.704 \\
\hline & D-MPNN & $0.762 \pm 0.065$ & 0.686 & 0.911 \\
\hline & GIN & $0.804 \pm 0.061$ & 0.738 & 0.911 \\
\hline & RF & $0.780 \pm 0.084$ & 0.699 & 0.890 \\
\hline & SVM & $0.740 \pm 0.068$ & 0.639 & 0.814 \\
\hline & KNN & $0.951 \pm 0.067$ & 0.801 & 1.003 \\
\hline & D-GIN cons. & $0.575 \pm 0.019$ & 0.548 & 0.622 \\
\hline & D-MPNN cons. & $0.647 \pm 0.028$ & 0.613 & 0.710 \\
\hline & GIN cons. & $0.666 \pm 0.029$ & 0.627 & 0.719 \\
\hline \multirow{9}{*}{$\log S$} & D-GIN & $0.867 \pm 0.070^{*}$ & 0.795 & 1.061 \\
\hline & D-MPNN & $0.896 \pm 0.030$ & 0.857 & 0.961 \\
\hline & GIN & $1.210 \pm 0.102$ & 1.088 & 1.400 \\
\hline & $\mathrm{RF}$ & $0.997 \pm 0.253$ & 0.760 & 1.284 \\
\hline & SVM & $1.006 \pm 0.154$ & 0.729 & 1.162 \\
\hline & KNN & $1.500 \pm 0.217$ & 1.057 & 1.676 \\
\hline & D-GIN cons. & $0.738 \pm 0.028$ & 0.705 & 0.820 \\
\hline & D-MPNN cons. & $0.762 \pm 0.012$ & 0.743 & 0.785 \\
\hline & GIN cons. & $0.881 \pm 0.045$ & 0.825 & 0.969 \\
\hline \multirow{9}{*}{$\log P$} & D-GIN & $0.529 \pm 0.064^{*}$ & 0.472 & 0.662 \\
\hline & D-MPNN & $0.600 \pm 0.063$ & 0.540 & 0.734 \\
\hline & GIN & $0.784 \pm 0.077$ & 0.716 & 0.901 \\
\hline & $\mathrm{RF}$ & $0.681 \pm 0.224$ & 0.470 & 0.928 \\
\hline & SVM & $0.693 \pm 0.134$ & 0.493 & 0.833 \\
\hline & KNN & $1.014 \pm 0.123$ & 0.743 & 1.102 \\
\hline & D-GIN cons. & $0.455 \pm 0.028$ & 0.428 & 0.515 \\
\hline & D-MPNN cons. & $0.475 \pm 0.027$ & 0.443 & 0.532 \\
\hline & GIN cons. & $0.566 \pm 0.034$ & 0.533 & 0.618 \\
\hline
\end{tabular}


Figure 2. LogD prediction results for all GNN and non-GNN model types. The different GNN architectures are colored in blue (D-GIN), orange (D MPNN) and green (GIN), the non-GNN architectures in gray (SVM), light orange (RF) and red (KNN) The average value of each model type independent of featurizations and training approaches is shown as a white dot and the median is shown as a dark gray line. The values are listed in tables 14 to 17 in the SI.

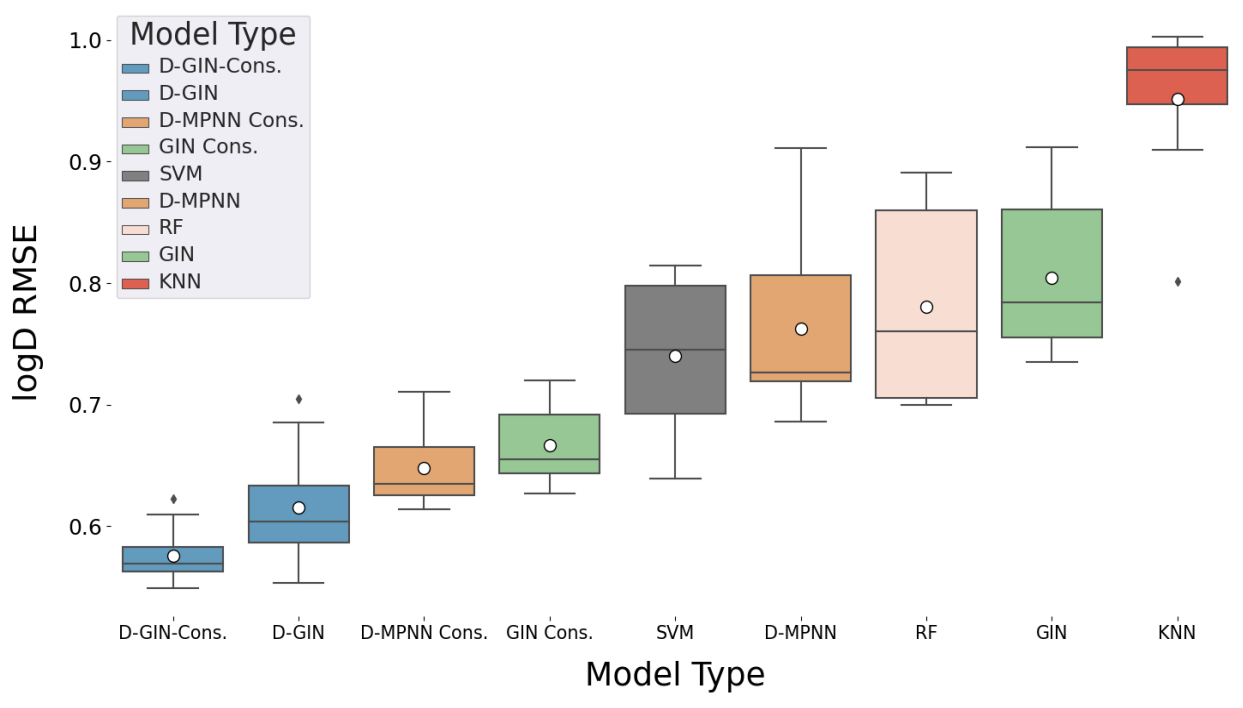

Figure 3. LogS prediction results for all GNN and non-GNN model types. The different GNN architectures are colored in blue (D-GIN), orange (D MPNN) and green (GIN), the non-GNN architectures in gray (SVM), light orange (RF) and red (KNN) The average value of each model type independent of featurizations and training approaches is shown as a white dot and the median is shown as a dark gray line. The values are listed in table tables 18 to 21 in the SI.

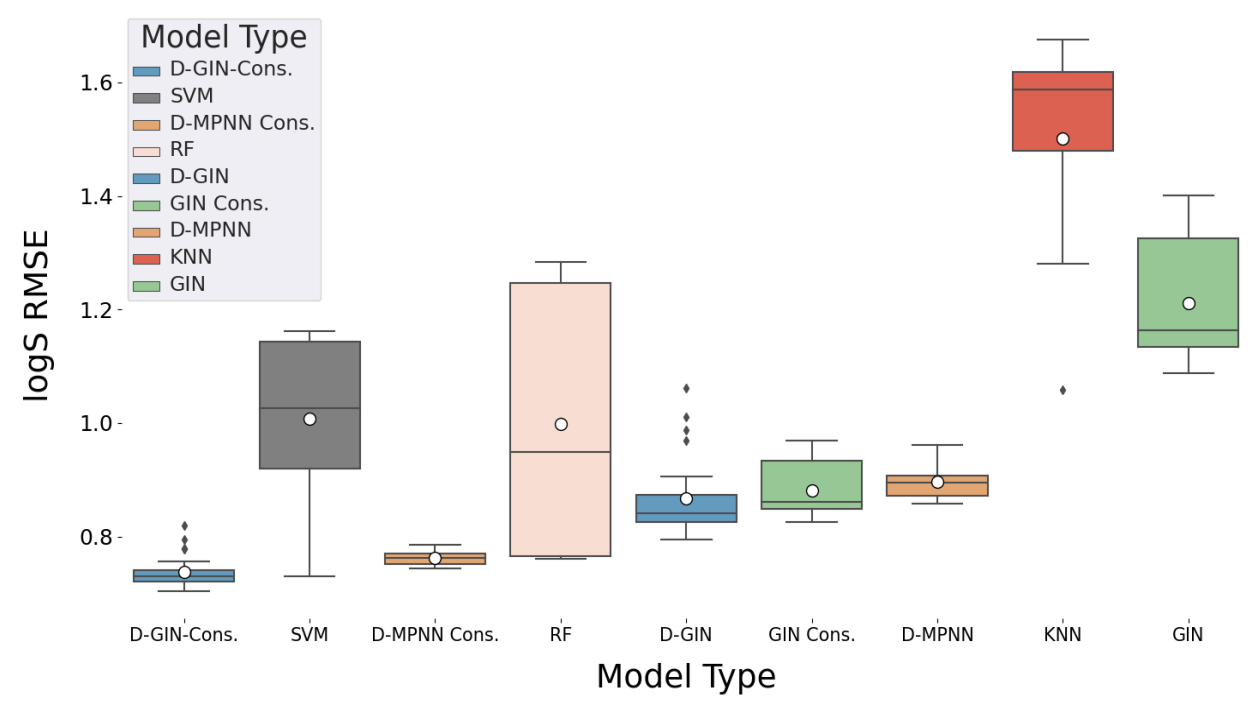


Figure 4. LogP prediction results for all GNN and non-GNN model types. The different GNN architectures are colored in blue (D-GIN), orange (D MPNN) and green (GIN), the non-GNN architectures in gray (SVM), light orange (RF) and red (KNN) The average value of each model type independent of featurizations and training approaches is shown as a white dot and the median is shown as a dark gray line. The values are listed in table tables 22 to 25 in the SI.

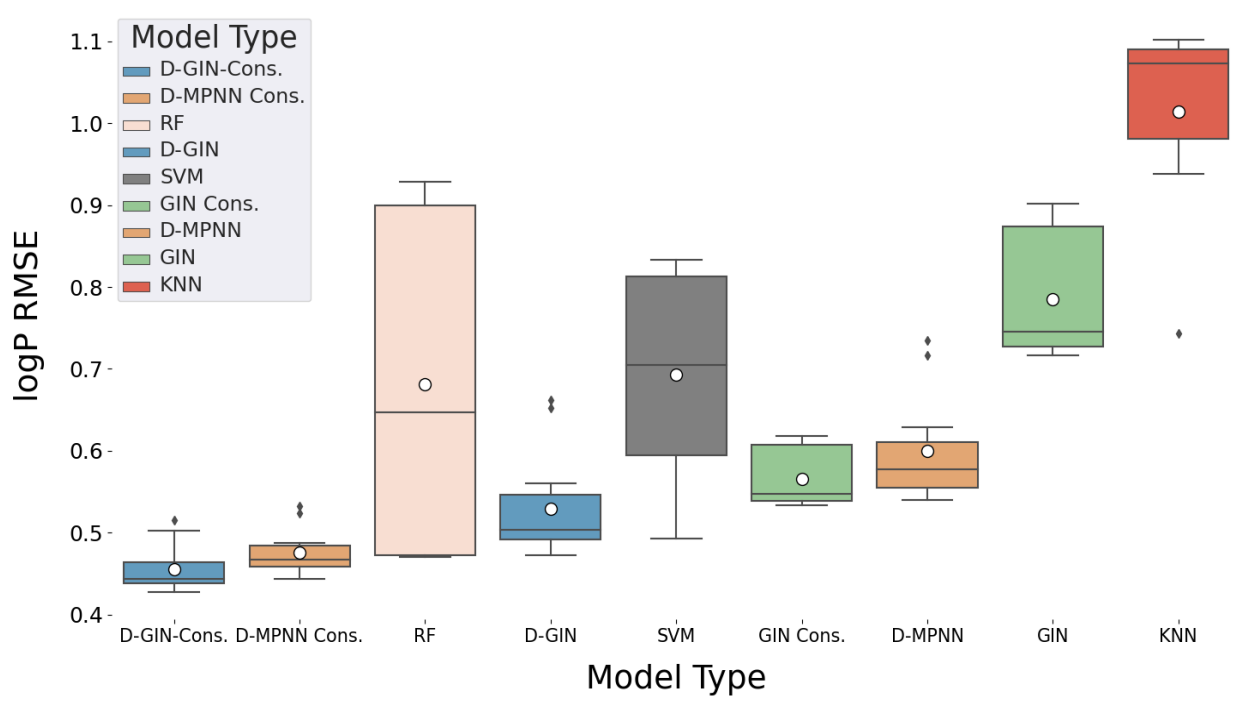

For the $\log \mathrm{D}$ property table 9 and figure 2 show that the D-GIN and its consensus types perform best with a mean, minimum and maximum $\log \mathrm{D}$ RMSE of 0.6150 .039 , 0.553 and 0.7048 , as well as for the corresponding consensus model with 0.5750 .0192 , 0.548 and 0.622. Even the consensus GIN performs on average (logD RMSE of 0.6660 .029 ) better than the best non-GNN method with a mean logD RMSE of 0.7400 .068 . For the $\log S$ prediction table 9 and figure 3 show that the best model type is the D-GIN consensus model type with an average RMSE of 0.738 0.028. It performs better than the best nonGNN model type (SVM), which gives the best minimum result without consensus (logS RMSE of 0.729) but its mean RMSE is higher at 1.006 0.154. The consensus D-MPNN also outperforms the D-GIN. For the $\log P$ property prediction, as can be seen in figure 4 and table 9, the consensus D-GIN (RMSE of 0.455 0.028) and consensus D-MPNN (RMSE of $0.4750 .027)$ show the best average performance. The RF and SVM model types give a low minimum RMSE value of 0.470 and 0.493 but their average RMSE of 0.6810 .224 and 0.6930 .134 is higher than all GNN model types except the GIN. Further investigations are required to give a rationale of why the consensus types perform always better than their individual counterparts. Furthermore it should be noted that a direct comparison between the average performance of the GNNs and non-GNNs can be misleading as both use different featurizations. The difference in the non-GNN's features is arguably higher than those in the GNNs and furthermore use 8 different featurization types compared to 7 in the case of GNNs, which also might lead to this high variance.

Figures 5 to 7 underline the results above and show the best results for each model and for each property. Each plot shows the average RMSE for the corresponding log unit on the right side with its $95 \%$ confidence interval (CI). The $r^{2}$ is displayed as a line on the right $\mathrm{x}$-axis. Additionally, the kernel densities for each model are included on the left side. Each bar on the $\mathrm{x}$-axis represents a model with different training and featurization strategies. The name and strategy used for each model is shown in the SI. It should be noted that for the $\log \mathrm{D}$ property, the best D-GIN models' CI is not out of the CI of either the D-MPNN or the GIN. Furthermore, for the logS property, the CI of the best D-GIN model lies within the best D-MPNN's CI but outside of the best GIN which is also true for the $\log P$ values. 
Overall these plots show that despite which physical property is predicted, the D-GIN model type (blue) performs better than the D-MPNN (orange) or the GIN (green).

Figure 5. $\log \mathrm{D}$ prediction results for each GNN model instance. The left y-axis specifies the $\log \mathrm{D}$ RMSE and the right, secondary $\mathrm{x}$-axis the corresponding $r^{2}$ values for each GNN model. D-GIN is colored blue, D-MPNN orange and GIN green. Each of the bars represent a different trained model a detailed description can be found in figure A1 in the SI. The accumulated kernel density for each model type is shown on the very left side. The red lines correspond to the $95 \%$ confidence intervals. The model names are a combination of model type (D-GIN, GIN, D-MPNN), training approach and featurization type - a detailed description of each model name can be found in tables tables 14 to 25 in the SI.

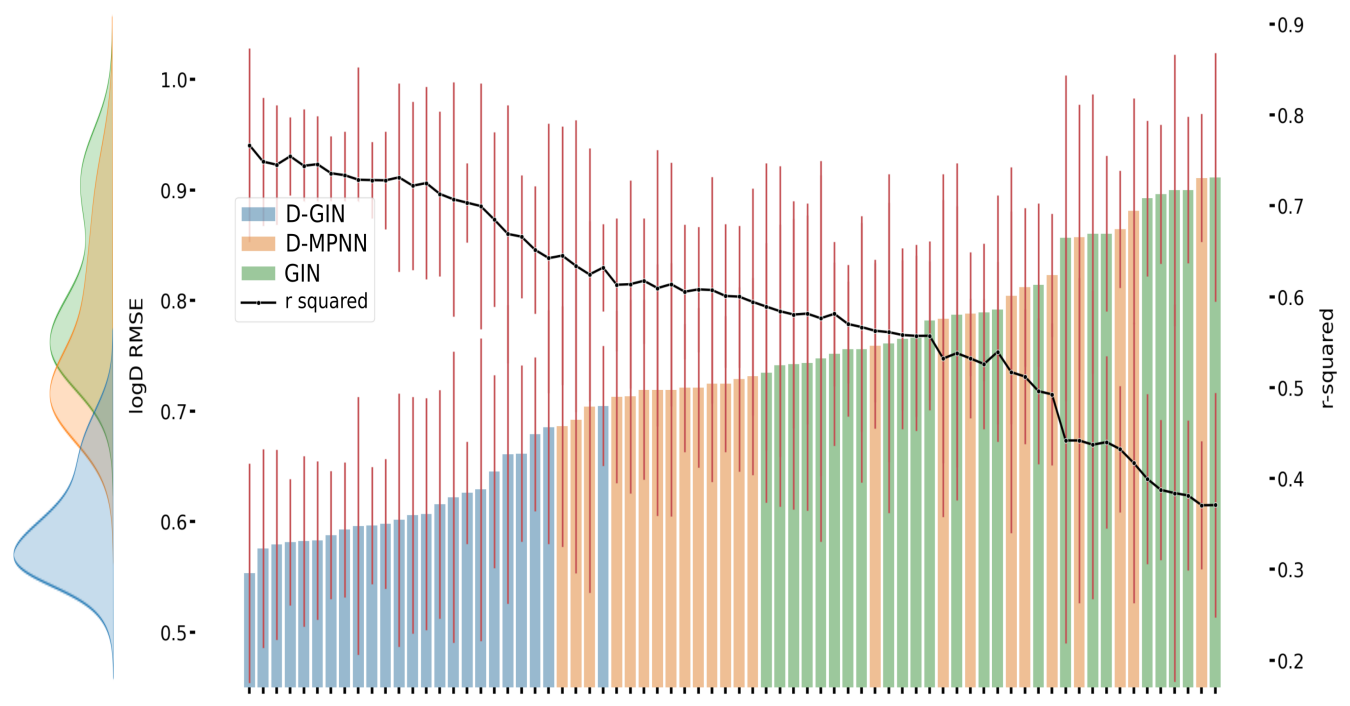


Figure 6. LogS prediction results for each GNN model instance. The left y-axis specifies the logS RMSE and the right, secondary $\mathrm{x}$-axis the corresponding $r^{2}$ values for each GNN model. D-GIN is colored blue, D-MPNN orange and GIN green. Each of the bars represent a different trained model a detailed description can be found in figure A3 in the SI. The accumulated kernel density for each model type is shown on the very left side. The red lines correspond to the $95 \%$ confidence intervals. The model names are a combination of model type (D-GIN, GIN, D-MPNN), training approach and featurization type - a detailed description of each model name can be found in tables tables 14 to 25 in the SI.

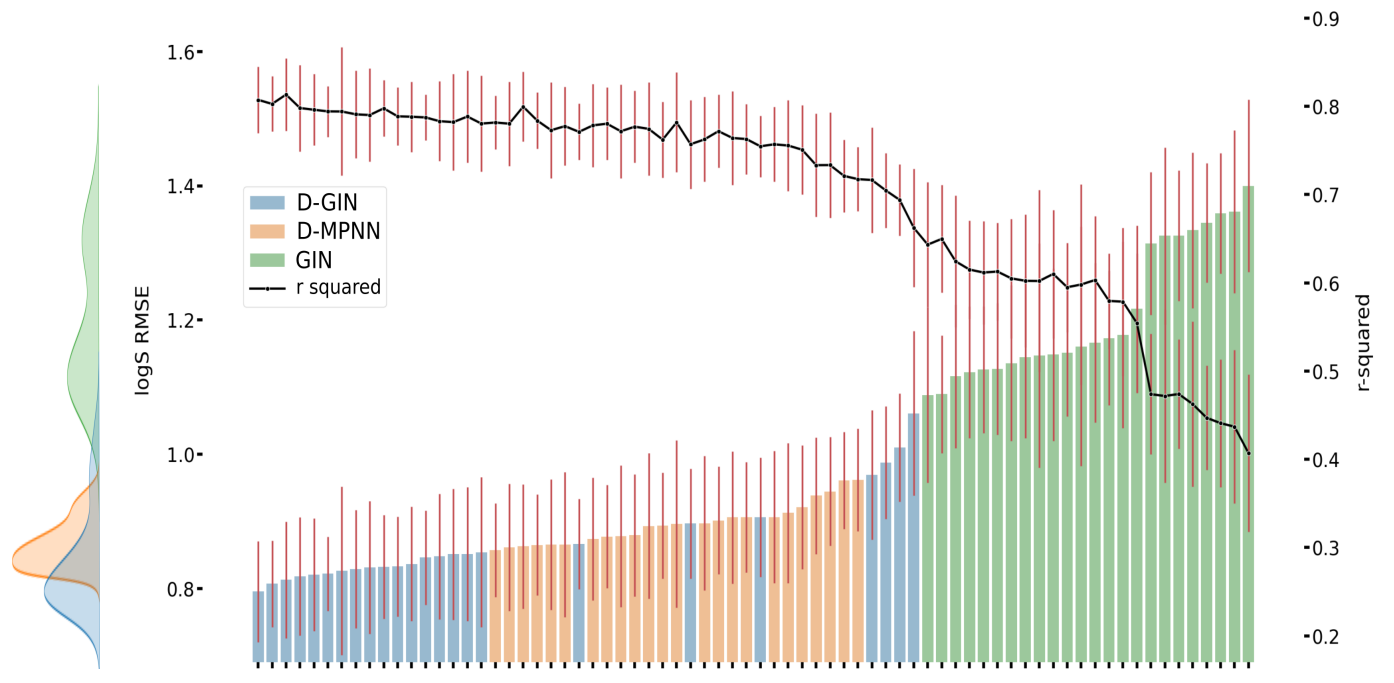

Figure 7. LogP prediction results for each GNN model instance. The left y-axis specifies the $\log P$ RMSE and the right, secondary $\mathrm{x}$-axis the corresponding $r^{2}$ values for each GNN model. D-GIN is colored blue, D-MPNN orange and GIN green. Each of the bars represent a different trained model a detailed description can be found in figure A2 in the SI. The accumulated kernel density for each model type is shown on the very left side. The red lines correspond to the $95 \%$ confidence intervals. The model names are a combination of model type (D-GIN, GIN, D-MPNN), training approach and featurization type - a detailed description of each model name can be found in tables tables 14 to 25 in the SI.

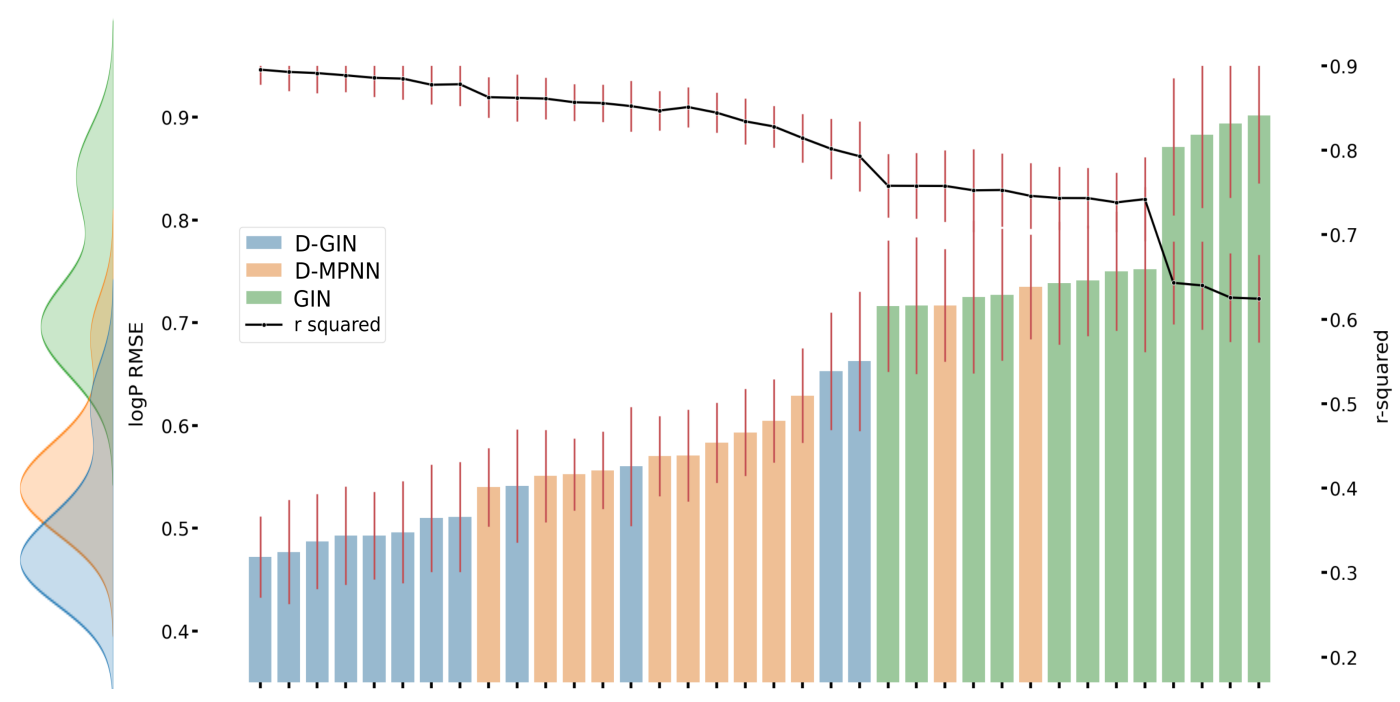


The reason why the D-GIN outperforms the GIN and D-MPNN in not only the best model but also on average by using different training and featurization strategies could be due to its higher complexity and depth. It uses the key aspects of both sub-models and might be able to abstract higher order features. This could be facilitated by including skip connections between edge feature extraction mainly done in the first part (D-MPNN) and node feature extraction in the second (GIN).

\subsection{Impact of molecular featurization and training strategies}

\subsubsection{Featurization strategies}

The mean performance of each featurization strategy across all model types and training strategies is shown in table 10. Considering all physical properties, featurization 5 is the worst performing featurization strategy with a mean $\log \mathrm{D}$ RMSE of 0.8130 .099 , a mean $\log S$ RMSE of 1.0990 .180 and a mean logP RMSE of 0.7600 .110 . This trend is also present when additionally separating according to the model type (Table 11 and figures 8 , 9 and 10). The reason for the general bad performance of featurization 5 might be that it only includes two node properties (atom type and formal charge - table 4) as well as one edge feature type (bond order - table 4). Featurization 6 (Table 5) also shows considerable bad performance when applied in combination with the GIN, where the average RMSE performance for the $\log \mathrm{D}$ and $\log S$ properties are worse than with featurization 5 . One explanation could be that the GIN utilizes node features quite extensively and featurization 6 only includes two node feature types similar to featurization 5 . The additional edge features in 6 without the appropriate architecture to deal with could confuse the GIN network rather than help with the property prediction.

Figure 8. LogD prediction results for all GNN model types depending on the featurization used (see section Molecular featurization). The mean is shown as a white dot whereas the median is shown as a dark gray line. Exact values are listed in table tables 14 to 17 in the SI.

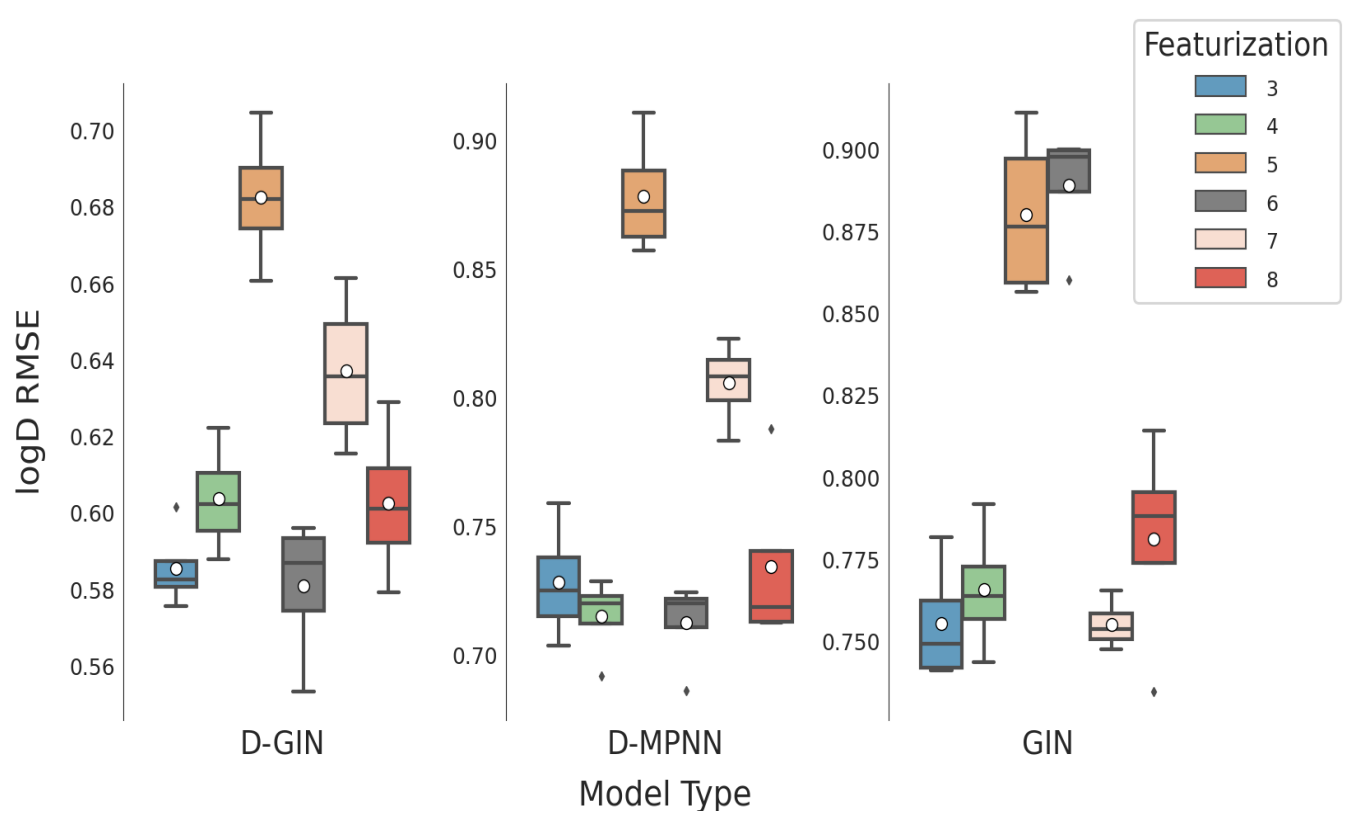




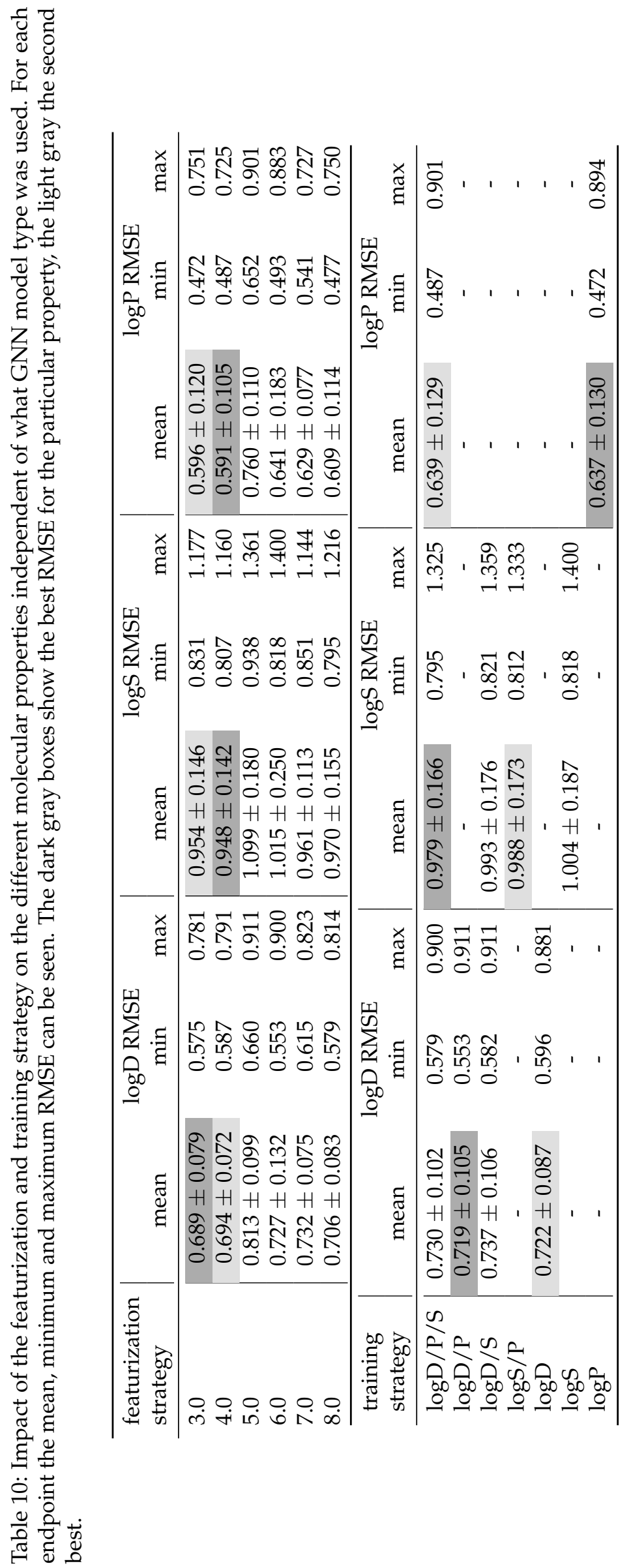


Figure 9. LogS prediction results for all GNN model types depending on the featurization used (see section Molecular featurization). The mean is shown as a white dot whereas the median is shown as a dark gray line. Exact values are listed in table tables 18 to 21 in the SI.

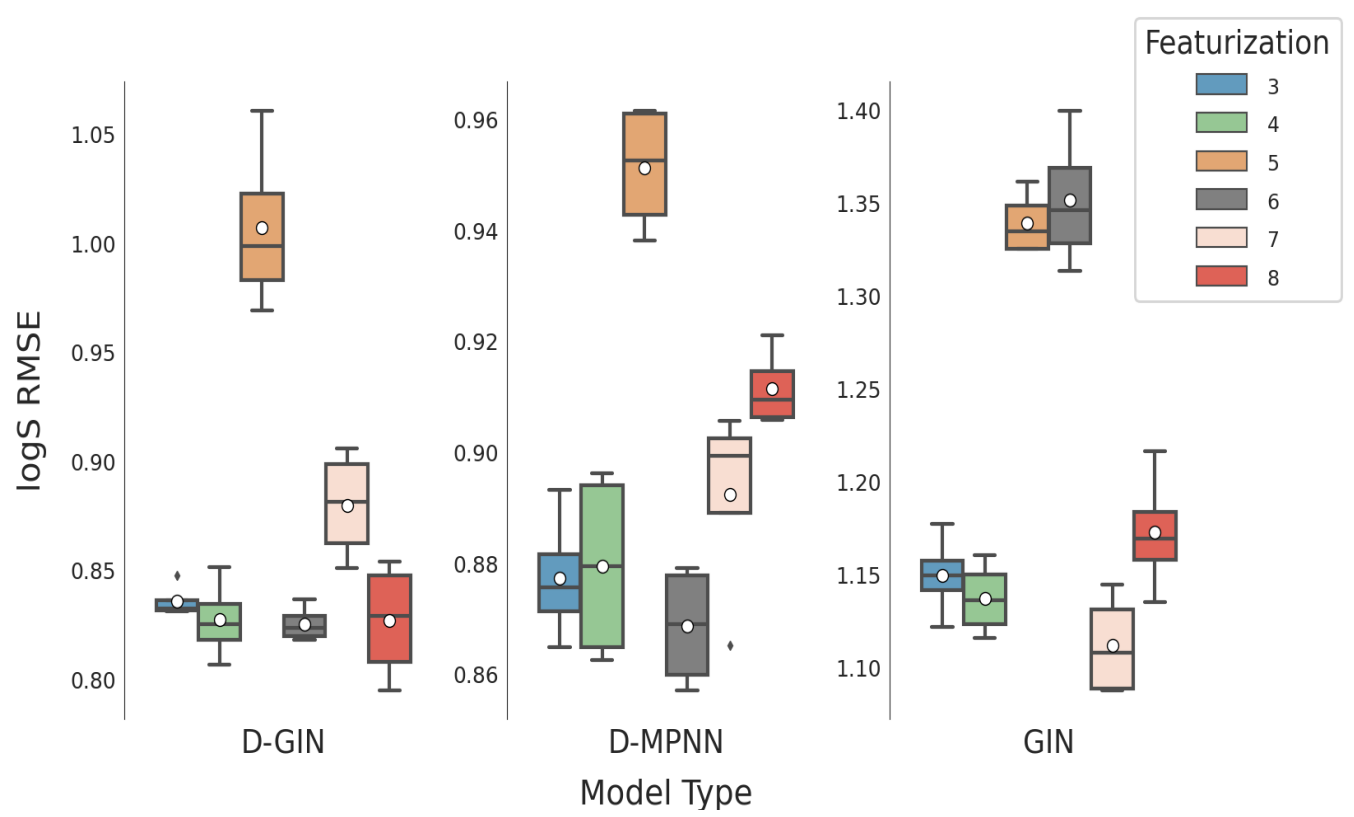

Figure 10. LogP prediction results for all GNN model types depending on the featurization used (see section Molecular featurization). The mean is shown as a white dot whereas the median is shown as a dark gray line. Exact values are listed in table tables 22 to 25 in the SI.

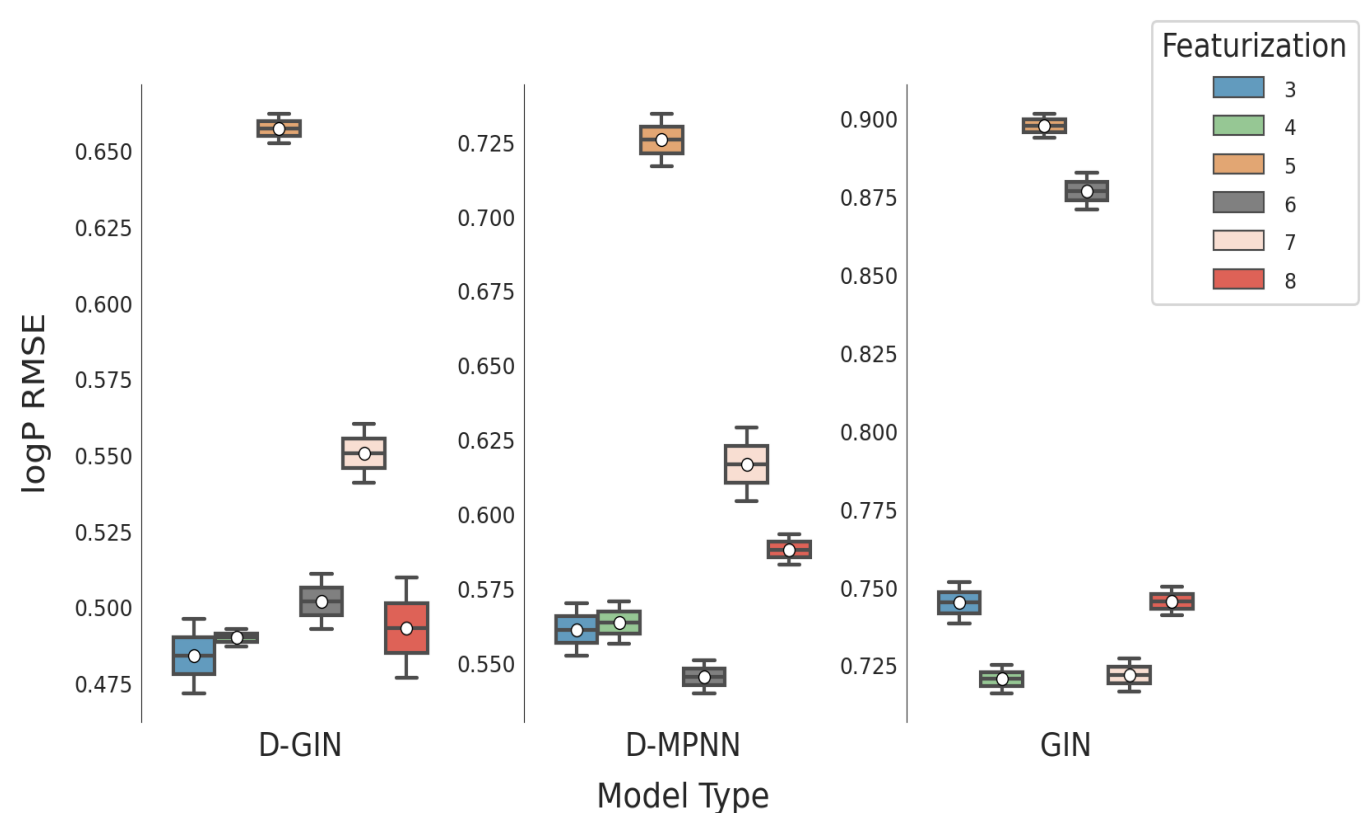

A tendency towards the best featurization strategy cannot as easily be observed as towards the worst featurization strategy described above. The mean RMSE across all training strategies and model types in table 10 show that featurization 3 and 4 (table 2 and 3) achieve the best results for $\log \mathrm{D}$ with a RMSE 0.6890 .079 and 0.6940 .072 , for $\operatorname{logS}$ with a RMSE 0.9540 .146 and 0.9480 .142 and for $\log P$ with a RMSE 0.5960 .120 and 0.591 
0.105 respectively. Both featurization strategies use all node and edge features applied throughout this work. They only differ in the way the size of a ring system is represented. Featurization 3 uses a float value calculated by 1 divided by the size of the ring system whereas featurization 4 uses a one-hot encoding of ten instances $(0,3,4,5,6,7,8,9,10,11)$. Table 11 shows the mean log RMSE with respect to featurization and model type. When counting the results according to the featurization strategy that performs either best or second best for each physical property, featurization 3 with 2 models as best and 3 models as second best performers achieve a better ranking than featurization 4 with one model as best and 2 models as second best. Both strategies perform similarly well, which is surprising as float values in combination with one-hot can confuse a neural network. This seems to be not the case throughout this work. An explanation could either be that this feature is not important. This question was partly answered by applying featurization 8 (table 7). It uses a set of optimized node and edge features. This was done by using a trained model and then removing one node or edge feature at a time and observing the RMSE of the prediction. The five node features and the three edge features that had the biggest impact on the RMSE were then taken as the features. This approach revealed that the size of ring systems for the node features is important and was therefore included in 8 . As for why featurization 3 and 4 perform well despite that the ring system size is of importance might be due to the fact that single float values can be added to one-hot encodings without loss in predictive power. To fully understand these findings further investigations are needed. Nevertheless, featurization 8 achieves two times the second best performance but never the best one. It shows an average good performance, but not as good as featurization 3 or 4. The average RMSE of featurization 6 and 7 (table 6) in table 10 show worse results than for featurization 3 and 4 . When counting the results according to the featurization strategy that performs either best or second best for each physical property, the best featurization is 6. It is used in 4 of the best performing runs and once in a second best run. But it only performs well in combination with two GNN architectures (D-GIN and D-MPNN) and strongly underperformance with the GIN. The reason for this might be the same as described above - the D-GIN and D-MPNN architecture types use primarily edge features for their information propagation and featurization 6 provides these. It utilizes only two node feature types, potentially reducing the noise for the feature extraction to a minimum in this setting. On average featurization strategy 6 and 7 perform similarly well but when separating the results at a model type level, it becomes evident that there is a strong model architecture dependency, so it seems important to choose the features according to the architecture at hand. Furthermore, featurization 3 might not give as good predictions as with featurization 6 or 7 , nevertheless, when unsure which features to use, simply adding more features could be the safer option than using less. This can be backed by comparing featurization 3 or 4 to e.g. 6,7 or 8 .

When analyzing the results for the non-GNN models and their different featurizations, the mean RMSE variance is large in comparison to the GNN models. Moreover, in similar deep-learning surveys that predicted molecular properties predominantly fingerprints are being used. From table 1 as well as 2, 4 and 3 in the SI one can see that especially featurizations that not only utilize fingerprints but also heavily include descriptors are performing exceptionally well. We think that when comparing GNN models with nonGNN models, different featurizations should be taken into consideration when trying to show (deep-learning) method superiority.

\subsubsection{Training strategies}

When looking at the impact of the different training strategies, table 10 provides the results for the average log RMSE with respect to model type and training approach. The lowest average $\log \mathrm{D}$ RMSE can be obtained by a multi-task strategy that involves $\log \mathrm{D}$ and $\log \mathrm{P}$ values. This is similar to the best training strategy for the $\log S$ property, which is a multi-task approach including $\log S$ and $\log \mathrm{P}$ properties. As for the $\log \mathrm{P}$ property, the 


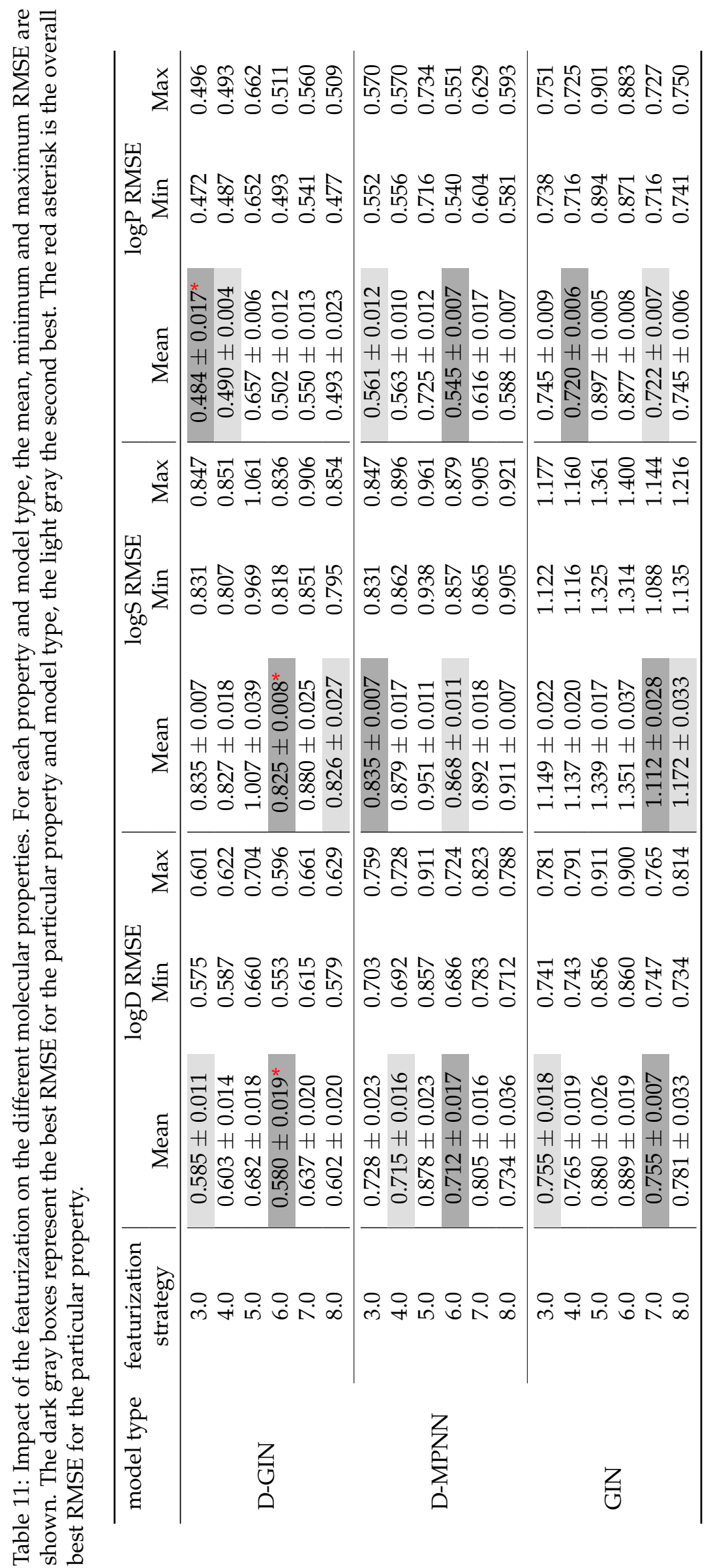


best approach is a single-task strategy including logP values but the multi-task approach which combines all physical properties achieves similarly good performance.

When analysing the log RMSE predictions with respect to training strategy and model type, table 10 and figures 8,9 and 10 show that there is no particularly favourable learning strategy for any of the model types. Most data sets are specific for one particular physical physical property (see section Molecular featurization). When comparing different learning strategies we thus focus on one particular physical property for each model type. Starting with the results for the prediction of the $\log \mathrm{D}$ property in table 4 , we can see that the overall best model (red asterisk) as well as the two best models for each model type (dark gray) are multi-task models. In particular the models with a combination of $\log \mathrm{D}$ and $\log \mathrm{P}$ properties perform well. When combining all models and featurizations, the best mean as well as minimum $\log \mathrm{D}$ RMSE is obtained by the $\log \mathrm{D} / \mathrm{P}$ training approach which results in values of 0.7190 .105 and 0.553 , respectively. This approach also results in the maximum $\log \mathrm{D}$ RMSE of 0.911 in comparison to a Single-Task learning strategy with $\log \mathrm{D}$ values only at a maximum of 0.881 and a minimum of $0.596 \log \mathrm{D}$ RMSE. The logS property also shows a tendency towards multi-task learning. The best model for this property is obtained by a strategy that includes all physical properties $(\log \mathrm{D}, \log \mathrm{S}$ and $\log \mathrm{P})$. This learning strategy most often includes the best model for each model type. When combining all model types, the best mean, minimum and maximum logS RMSE of $0.9790 .166,0.795$ and 1.325 respectively is obtained by using all properties during training.

Figure 11. LogD prediction results for all GNN model types according to the used training strategy. Blue box shows the performance of the training strategy using $\log \mathrm{D}, \log \mathrm{S}$ and $\log \mathrm{P}$ combined. The gray and orange box show the results utilizing a combination of $\log \mathrm{D}$ and $\log \mathrm{P}$ and $\log \mathrm{D}$ and $\log \mathrm{S}$ for training. The pale box shows the results using $\log \mathrm{D}$ for training. The mean is shown as a white dot whereas the median is shown as a dark gray line. Exact values are listed in table tables 14 to 17 in the SI.
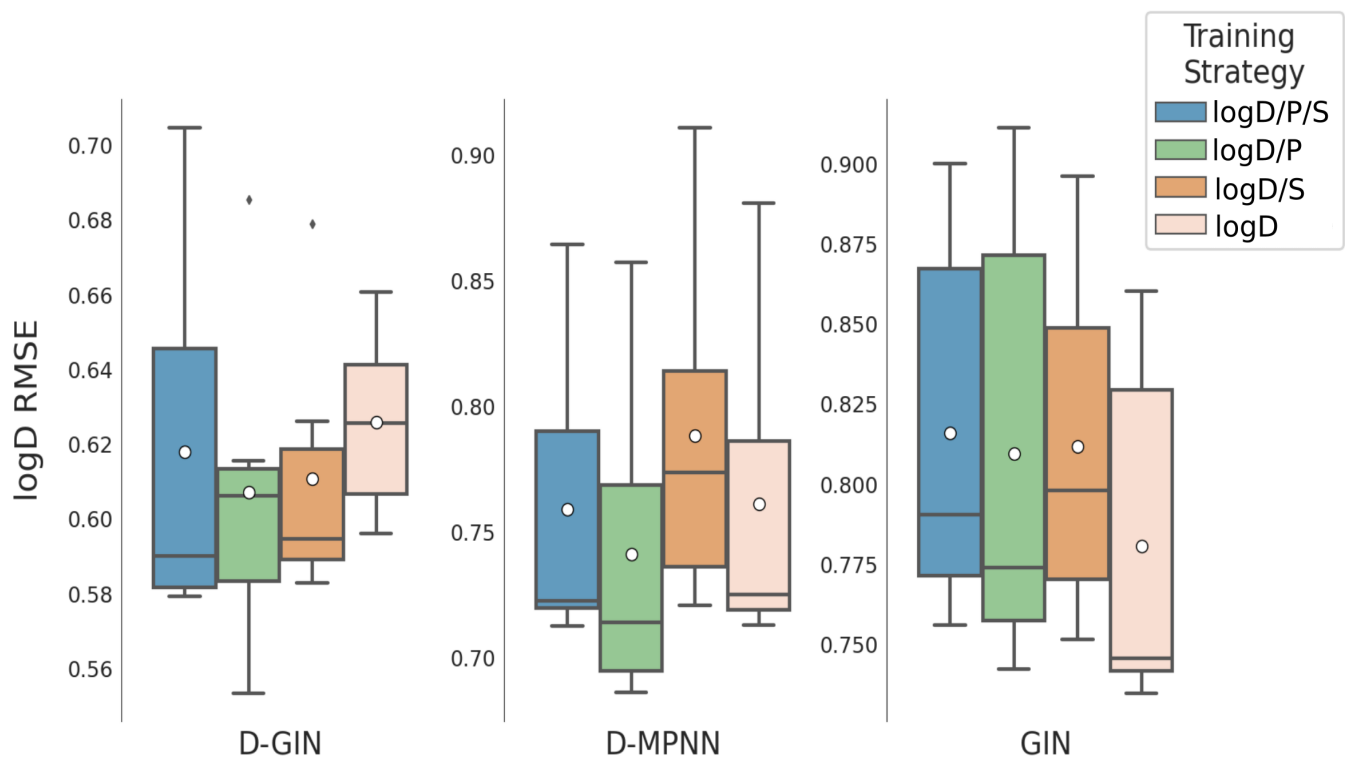

Model Type 
)

훙 항

政

to

동

e

은 흉

ร.

s

2.

密

\%.

몰

的

홍

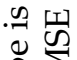

롱르

홍

E.

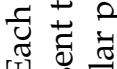

s.

을

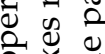

을ㅇㅇㅇ

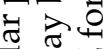

列品

흘 현

跣

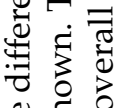

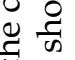

E

政

कo

E.

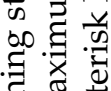

希

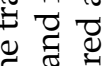

형 홑

敋

है

피 छ

ช ฮ ช

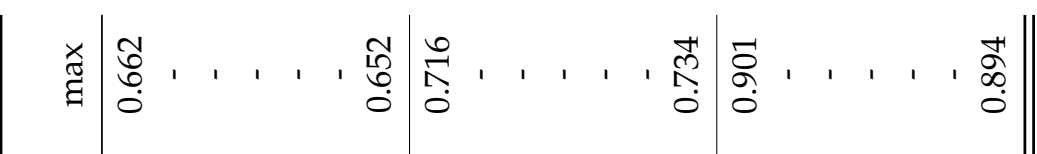

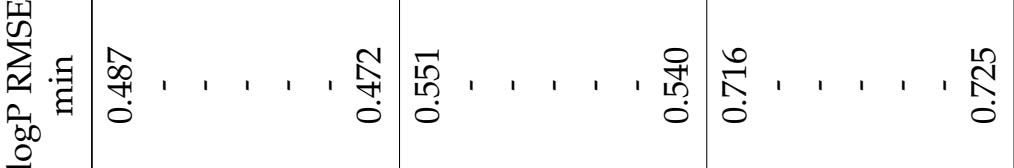

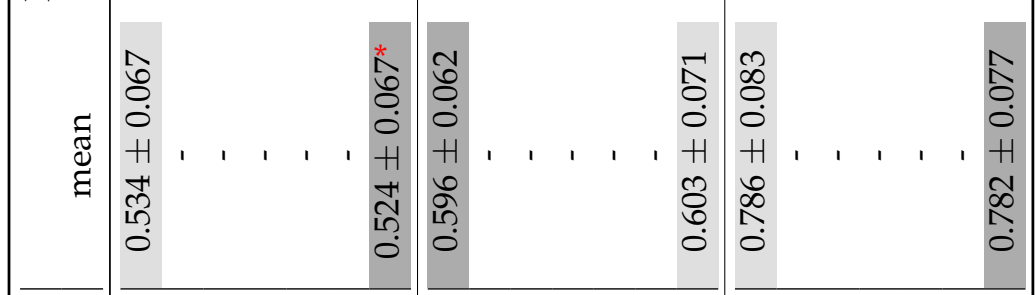

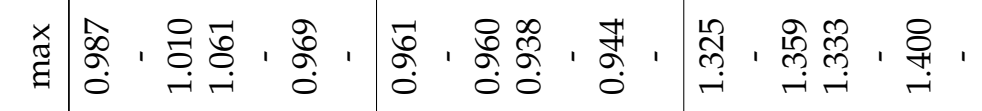

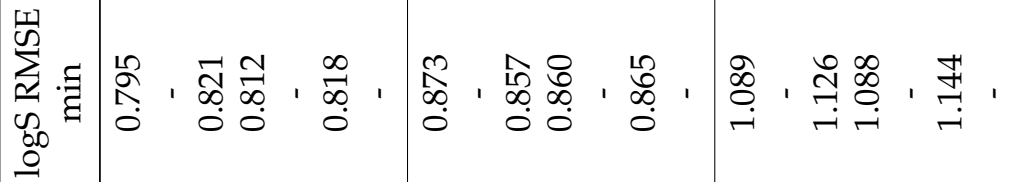

으

च

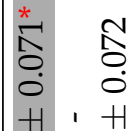

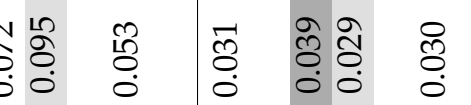

$\begin{array}{lll}10 & \infty \\ \infty & \infty \\ 0 & \infty \\ 0\end{array}$

N
0
0

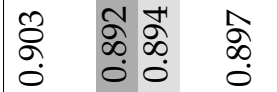

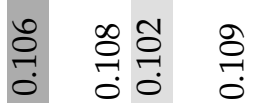

西

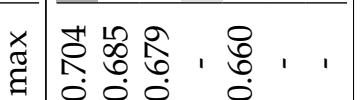

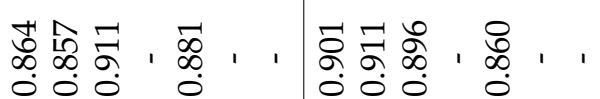

崖

6

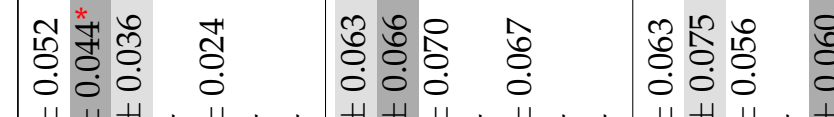

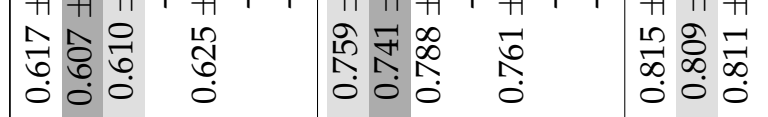

20

.

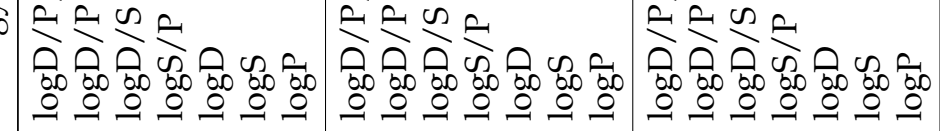

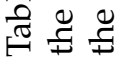

$\sum_{1}^{Z}$ 
Figure 12. LogS prediction results for all GNN model types according to the used training strategy. Blue box shows the performance of the training strategy using $\log \mathrm{D}, \log \mathrm{S}$ and $\log \mathrm{P}$ combined. The gray and orange box show the results utilizing a combination of $\log S$ and $\log \mathrm{P}$ and $\log \mathrm{D}$ and $\log \mathrm{S}$ properties respectively. The red box shows the results using logS for training. The mean is shown as a white dot whereas the median is shown as a dark gray line. Exact values are listed in table tables 18 to 21 in the SI.

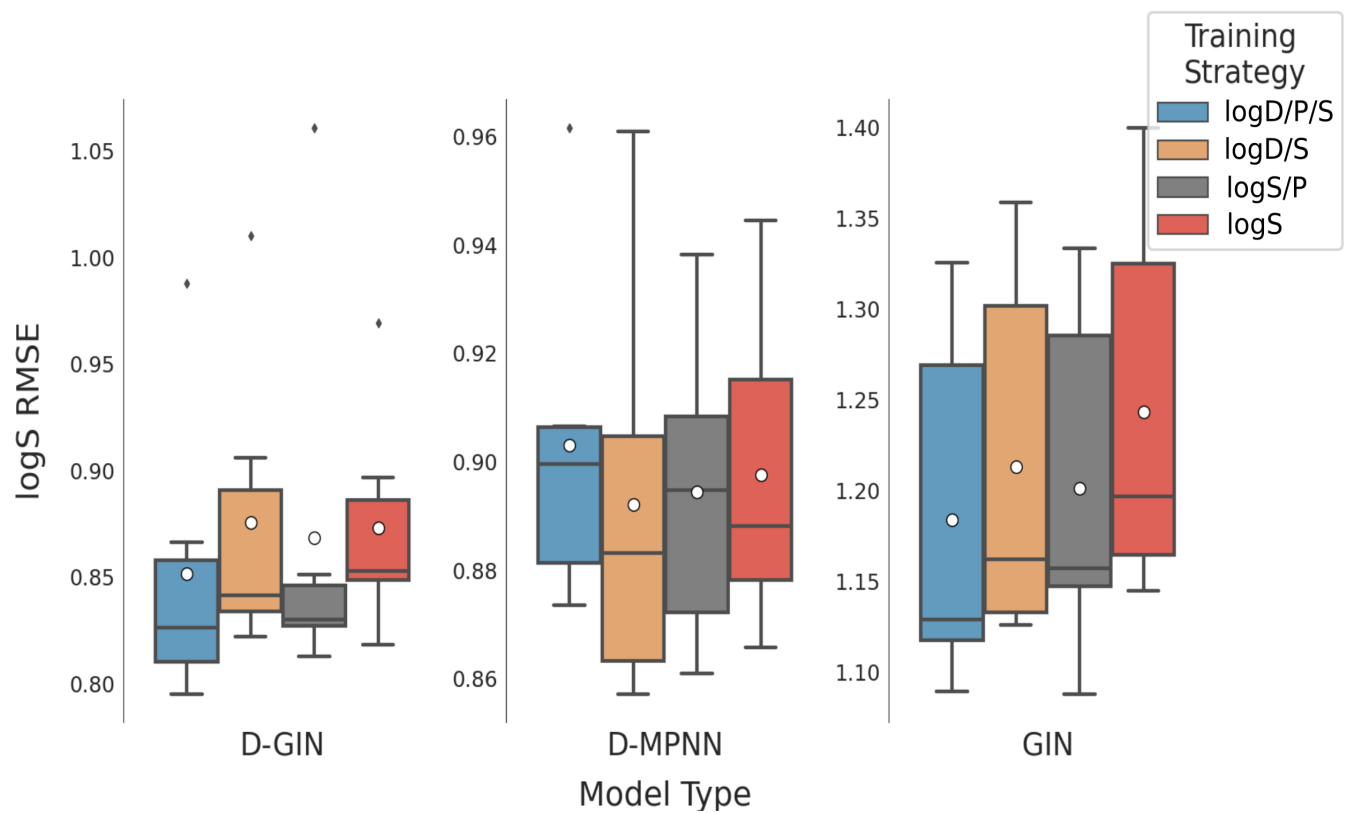

Figure 13. LogP prediction results for all GNN model types according to the used training strategy. Blue box shows the performance of the training strategy using $\log \mathrm{D}, \log \mathrm{S}$ and $\log \mathrm{P}$ combined. The dark orange box shows the results using $\log \mathrm{P}$ for training. The mean is shown as a white dot whereas the median is shown as a dark gray line. Exact values are listed in table tables 22 to 25 in the SI.

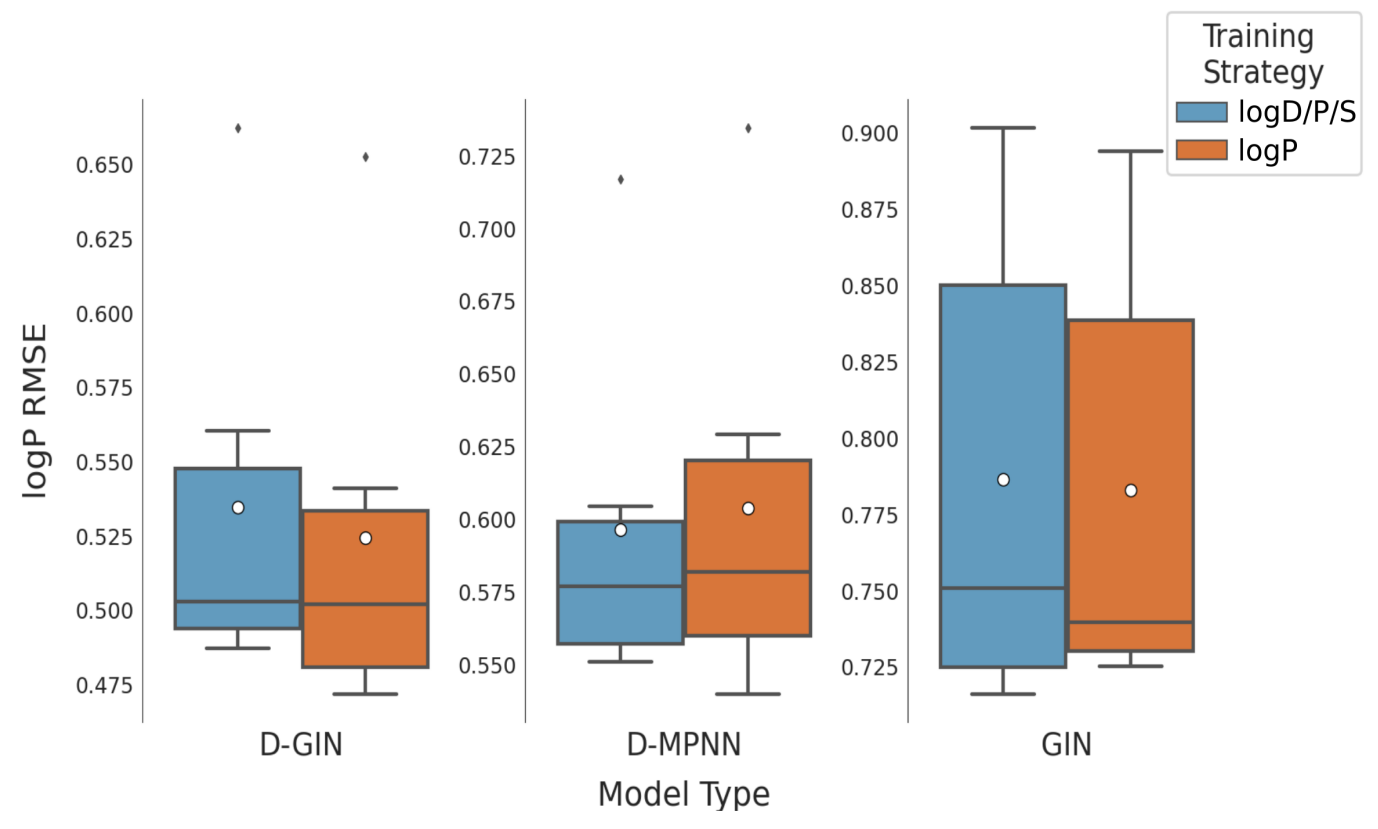




\section{Conclusions}

We introduced the directed-edge graph isomorphism network (D-GIN), a novel graph neural network that shows improved average performance for aqueous solubility and lipophilicity prediction to other baseline models and we argue that combining different models with distinct features can increase the depth of the model while also increasing its predictive power. Furthermore, we highlight that comparing single performance metrics as in table 1 can be misleading when trying to show model superiority for stochastic algorithms like neural networks. Data scarcity and the behaviour of optimizers like stochastic gradient descent can have an impact on the outcome and we argue that it is useful to utilize different training strategies and featurizations in order to obtain multiple results for the same model to compare and thus get more information regarding general, average model performance. Such an approach can help to gain insight in the behaviour of black box models such as GNNs and make them more useful in the future. In concurrence to previous publications ([34],[35],[36]) we showed that there is a tendency towards multitask learning approaches for the GNNs utilized in this survey. On average they performed better than their single-task counterpart for the corresponding physical property. We could not find clear evidence that more than two properties increase the model's performance. Furthermore, we highlighted that the usage of additional features did not improve the GNN model performance. It is necessary to be aware of the type of GNN that is used and whether its architecture focuses more on edge or node features. When trying to obtain the best performing model it can be advisable to do feature engineering, but when in doubt which features to use, it can be safer to use more than less. We showed that this awareness can help improve the GNNs predictive power at hand. Deep-learning model type comparisons with different featurizations or different data splits for training and evaluation should in general be looked at with caution as their results can vary substantially without even changing the architecture or its hyperparameters. For the non-GNN models we could conclude that by excessively adding descriptors to the molecular fingerprint the performance of these models increases substantially. We further argued that for future comparisons it would be advisable to include not only fingerprints but also descriptors to the non-GNN baseline models in order to be more competitive. By combining the best GNN model with the best non-GNN model we could see a slight improvement in the overall performance in all cases. Consensus models have often shown to improve the performance. However in this case further investigations are needed to get to a final conclusion on why this is the case.

Author Contributions: Conceptualization: OW, MW, TS and TL. Methodology: OW. Software: OW. Investigation: OW. Writing- Original Draft: OW. Writing-ReviewEditing: OW, MK, MW, TL and TS. Funding Acquisition: TL and MK. Resources: TL and MK. Supervision: TS, MW and TL. All authors have given approval to the final version of the manuscript.

Funding: This research received no external funding.

Institutional Review Board Statement: -

Informed Consent Statement: Informed consent was obtained from all subjects involved in the study.

Data Availability Statement: Python package used in this work (release v0.1):

https://github.com/spudlig/graph_networks

Data is available on https://zenodo.org/record/5137613.YQortyWxVhG

Acknowledgments: The authors thank Servier Research Institute (IDRS), Inte:Ligand GmbH and the University of Vienna for financial and advisory support.

Conflicts of Interest: There are no conflicts of interest or disclosures associated with this manuscript. 


\section{Appendix A}

The appendix includes informational materials that show the featurization of the non-GNN baseline models in table 13, in figures A1, A3 and A2 the individual models and their correpsonding names can be seen. These are the same figures as in the main body but include the unique identifiers. These identifiers show what kind of model type, featurization and training approch was used when looked up in tables tables 14 to 25 .

Table 13: Non-GNN featurization. The identifier is used as reference.

\begin{tabular}{c|lccc}
\hline identifier & fingerprint & radius & nr. bits & descriptor \\
\hline \hline 10 & ECFP & 4 & 1024 & No \\
11 & ECFP & 4 & 1536 & No \\
12 & ECFP & 4 & 2048 & No \\
13 & MACCSKeys & - & - & No \\
14 & ECFP & 4 & 1024 & Yes \\
15 & ECFP & 4 & 1536 & Yes \\
16 & ECFP & 4 & 2048 & Yes \\
17 & MACCSKeys & - & - & Yes \\
\hline \hline
\end{tabular}


Figure A1. The left y-axis specifies the logD RMSE and the right, secondary $x$-axis the corresponding $r^{2}$ values for each GNN model. The D-GIN is colored blue, the D-MPNN orange and the GIN green. Each of the boxes represents one different model run. The kernel density of each model is shown on the very left side. The red lines correspond to the $95 \%$ confidence intervals. The model names are a combination of model type (D-GIN, GIN, D-MPNN), training approach and featurization type - a detailed description of each model name can be found in tables tables 14 to 25 in the SI.

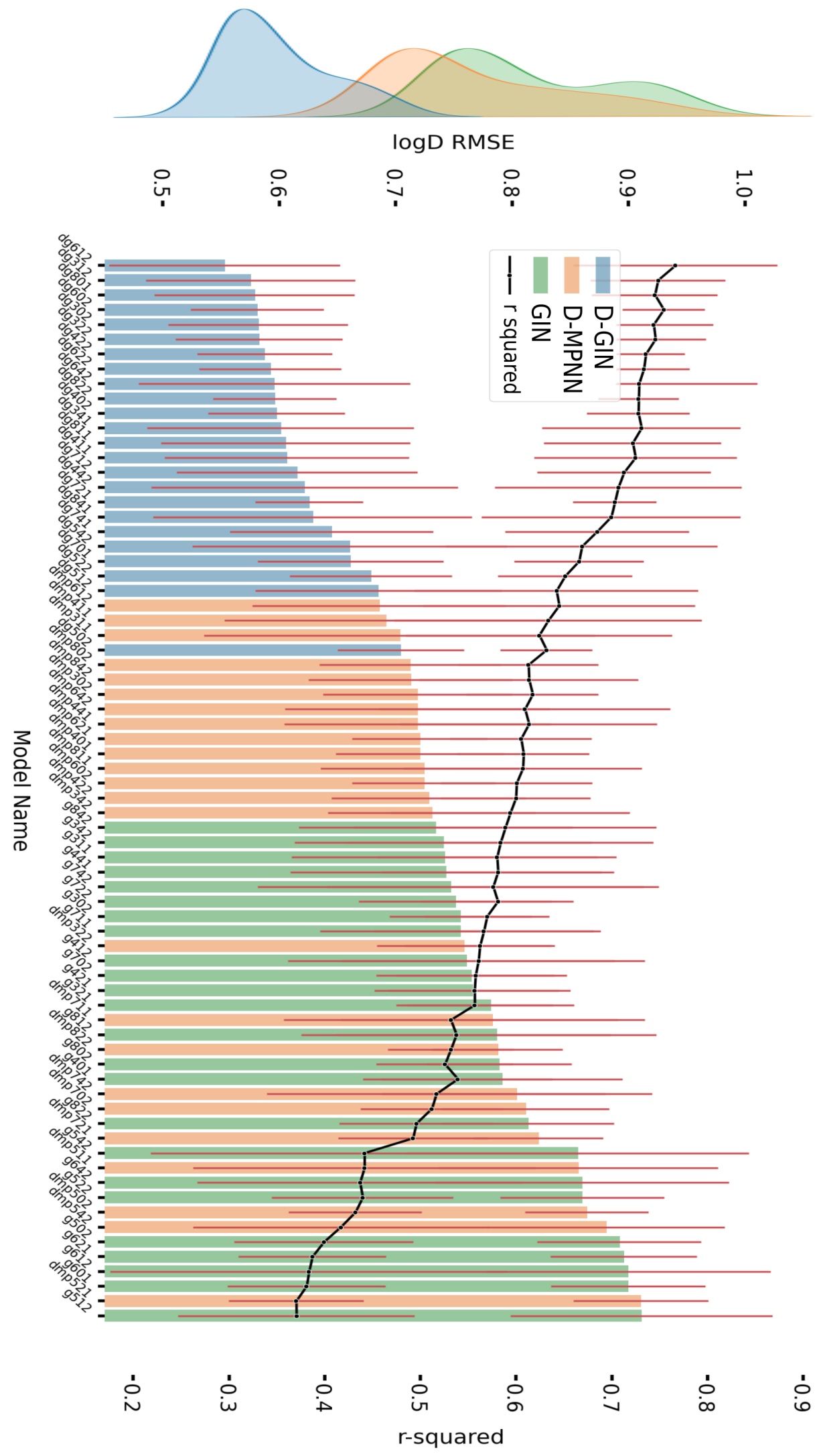


Figure A2. The left y-axis specifies the $\log P$ RMSE and the right, secondary $x$-axis the corresponding $r^{2}$ values for each GNN model. The D-GIN is colored blue, the D-MPNN orange and the GIN green. Each of the boxes represents one different model run. The kernel density of each model is shown on the very left side. The red lines correspond to the $95 \%$ confidence intervals. The model names are a combination of model type (D-GIN, GIN, D-MPNN), training approach and featurization type - a detailed description of each model name can be found in tables tables 14 to 25 in the SI.
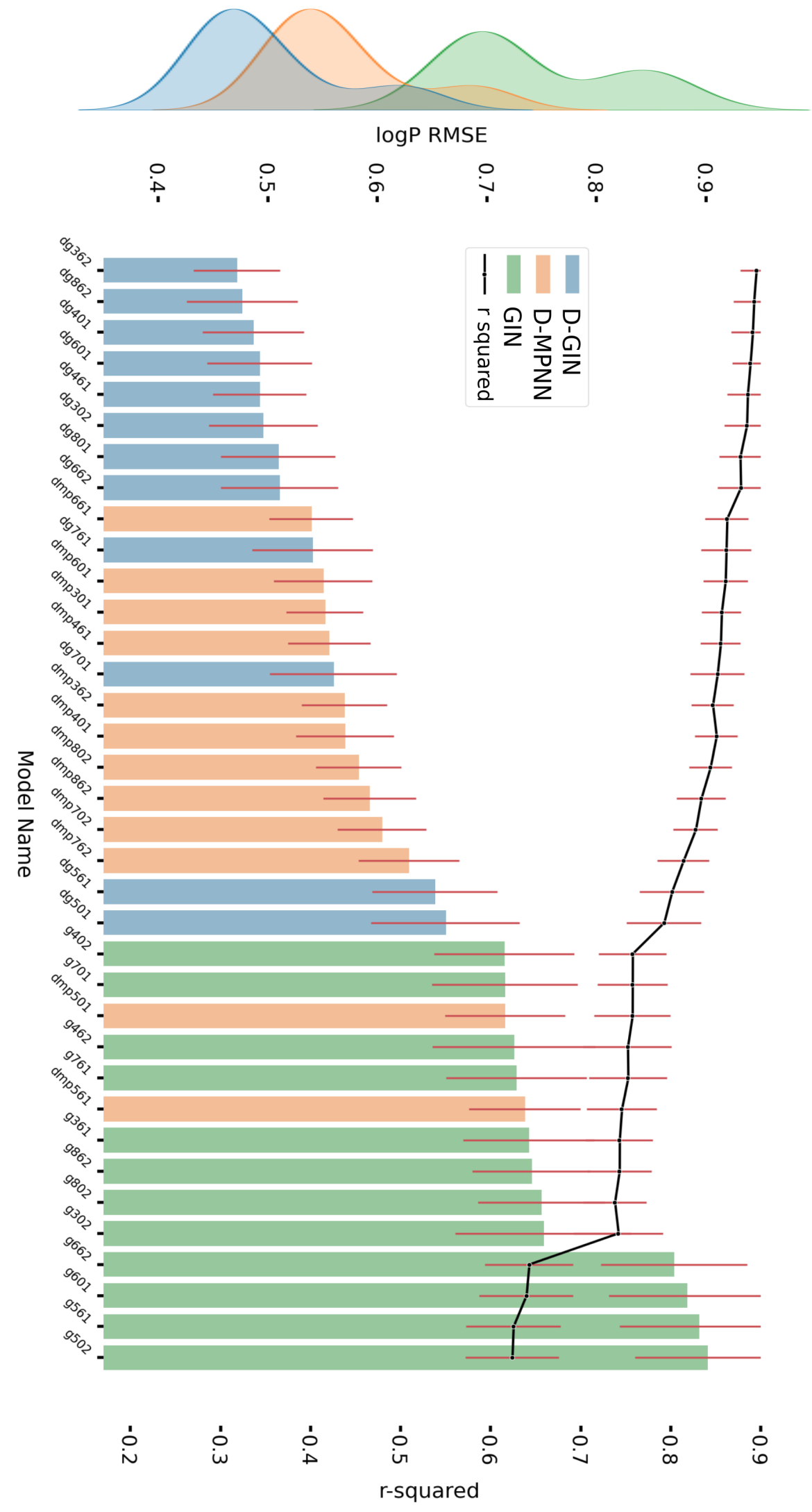
Figure A3. The left y-axis specifies the logS RMSE and the right, secondary $x$-axis the corresponding $r^{2}$ values for each GNN model. The D-GIN is colored blue, the D-MPNN orange and the GIN green. Each of the boxes represents one different model run. The kernel density of each model is shown on the very left side. The red lines correspond to the $95 \%$ confidence intervals. The model names are a combination of model type (D-GIN, GIN, D-MPNN), training approach and featurization type - a detailed description of each model name can be found in tables tables 14 to 25 in the SI.
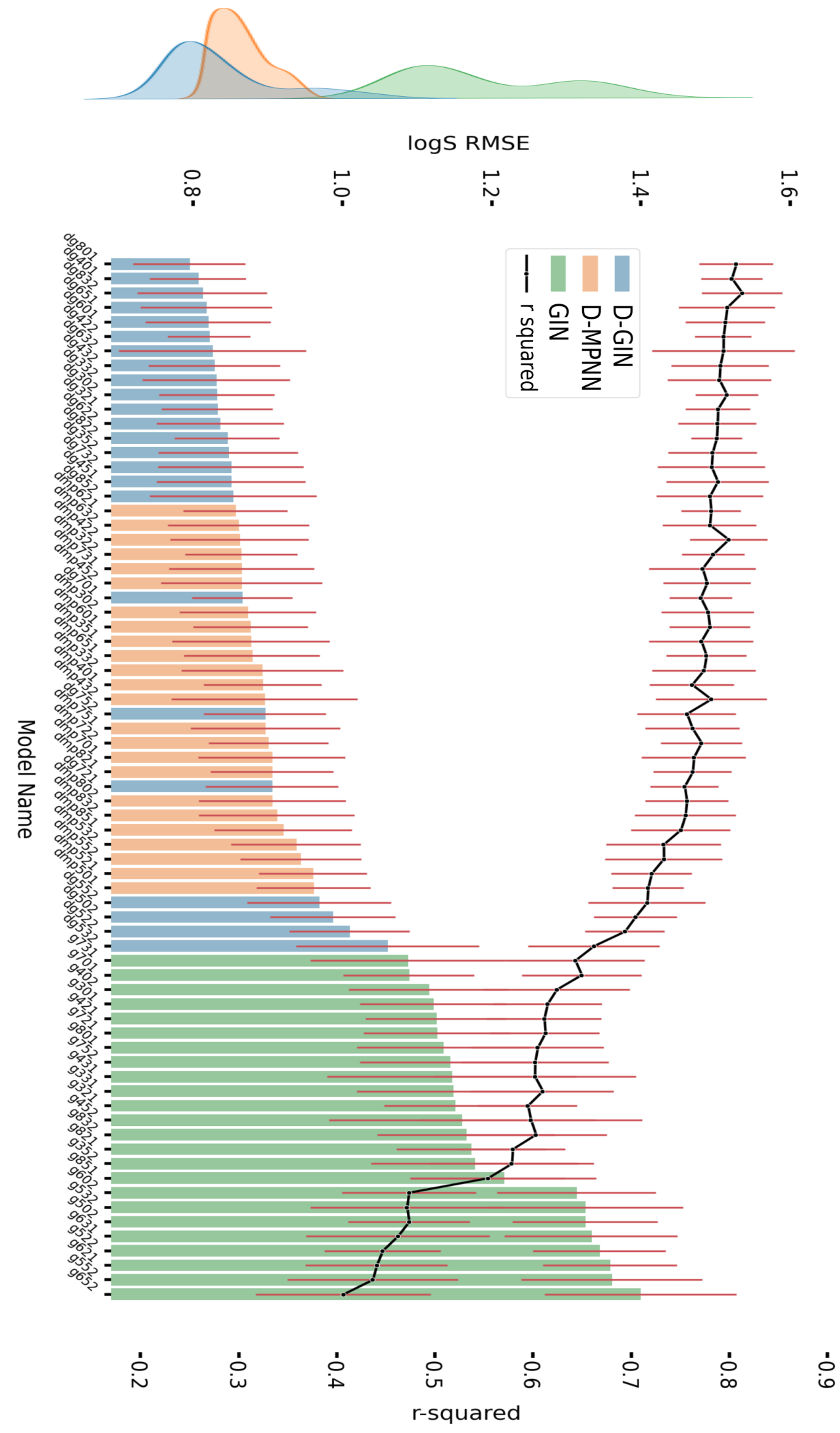
Table 14: Shows the $\log$ D RMSE and $r^{2}$ results for the D-GIN model type used during this survey. The last column consist of the unique identify. Training strategy 0 represents a training strategy combining $\log \mathrm{D}, \log \mathrm{S}$ and $\log \mathrm{P}, 1$ represents a strategy combining $\log \mathrm{D}$ and $\log \mathrm{P}, 2$ stands for a combination of $\log \mathrm{D}$ and $\log \mathrm{S}, 3$ represents a strategy using $\log \mathrm{S}$ and $\log \mathrm{P}, 4$ represents a strategy using $\log \mathrm{D}, 5$ represents a strategy using $\log \mathrm{S}$ and 6 represents a strategy using $\log \mathrm{P}$.

\begin{tabular}{|c|c|c|c|c|c|}
\hline $\begin{array}{l}\text { model } \\
\text { type }\end{array}$ & $\begin{array}{l}\text { training } \\
\text { strategy }\end{array}$ & $\begin{array}{l}\text { featurization } \\
\text { strategy }\end{array}$ & $\begin{array}{l}\log \mathrm{D} \\
\text { RMSE }\end{array}$ & $r^{2}$ & $\begin{array}{l}\text { unique } \\
\text { ID }\end{array}$ \\
\hline D-GIN & 2 & 5 & $0.679 \pm 0.034$ & $0.652 \pm 0.035$ & $\operatorname{dg} 522$ \\
\hline D-GIN & 2 & 8 & $0.596 \pm 0.026$ & $0.728 \pm 0.021$ & dg822 \\
\hline D-GIN & 2 & 4 & $0.587 \pm 0.029$ & $0.736 \pm 0.020$ & $\operatorname{dg} 422$ \\
\hline D-GIN & 2 & 3 & $0.582 \pm 0.035$ & $0.746 \pm 0.027$ & dg322 \\
\hline D-GIN & 0 & 3 & $0.582 \pm 0.038$ & $0.744 \pm 0.031$ & dg302 \\
\hline D-GIN & 0 & 6 & $0.581 \pm 0.028$ & $0.755 \pm 0.021$ & $\operatorname{dg} 602$ \\
\hline D-GIN & 0 & 4 & $0.598 \pm 0.029$ & $0.728 \pm 0.027$ & $\operatorname{dg} 402$ \\
\hline D-GIN & 4 & 3 & $0.601 \pm 0.057$ & $0.731 \pm 0.052$ & dg341 \\
\hline D-GIN & 0 & 8 & $0.579 \pm 0.043$ & $0.745 \pm 0.033$ & dg801 \\
\hline D-GIN & 1 & 3 & $0.575 \pm 0.044$ & $0.749 \pm 0.035$ & dg312 \\
\hline D-GIN & 1 & 8 & $0.605 \pm 0.053$ & $0.722 \pm 0.046$ & dg811 \\
\hline D-GIN & 4 & 6 & $0.596 \pm 0.058$ & $0.729 \pm 0.062$ & $\operatorname{dg} 642$ \\
\hline D-GIN & 1 & 4 & $0.606 \pm 0.052$ & $0.725 \pm 0.053$ & $\operatorname{dg} 411$ \\
\hline D-GIN & 1 & 7 & $0.615 \pm 0.051$ & $0.713 \pm 0.045$ & dg712 \\
\hline D-GIN & 4 & 4 & $0.622 \pm 0.065$ & $0.707 \pm 0.065$ & $\operatorname{dg} 442$ \\
\hline D-GIN & 2 & 7 & $0.626 \pm 0.023$ & $0.704 \pm 0.022$ & dg721 \\
\hline D-GIN & 4 & 8 & $0.629 \pm 0.068$ & $0.700 \pm 0.068$ & dg841 \\
\hline D-GIN & 4 & 7 & $0.645 \pm 0.043$ & $0.685 \pm 0.048$ & dg741 \\
\hline D-GIN & 4 & 5 & $0.660 \pm 0.067$ & $0.669 \pm 0.071$ & dg542 \\
\hline D-GIN & 0 & 7 & $0.661 \pm 0.039$ & $0.666 \pm 0.034$ & dg701 \\
\hline D-GIN & 1 & 5 & $0.685 \pm 0.052$ & $0.643 \pm 0.074$ & dg512 \\
\hline D-GIN & 1 & 6 & $0.553 \pm 0.049$ & $0.767 \pm 0.053$ & $\operatorname{dg} 612$ \\
\hline D-GIN & 0 & 5 & $0.704 \pm 0.027$ & $0.632 \pm 0.024$ & dg502 \\
\hline D-GIN & 2 & 6 & $0.592 \pm 0.030$ & $0.734 \pm 0.024$ & $\operatorname{dg} 622$ \\
\hline D-GIN cons. & 2 & 5 & $0.605 \pm 0.032$ & $0.719 \pm 0.031$ & dg522_cons \\
\hline D-GIN cons. & 0 & 5 & $0.622 \pm 0.030$ & $0.704 \pm 0.027$ & dg502_cons \\
\hline D-GIN cons. & 0 & 7 & $0.603 \pm 0.030$ & $0.722 \pm 0.025$ & dg701_cons \\
\hline D-GIN cons. & 1 & 5 & $0.609 \pm 0.056$ & $0.714 \pm 0.062$ & dg512_cons \\
\hline D-GIN cons. & 1 & 6 & $0.548 \pm 0.051$ & $0.769 \pm 0.049$ & dg612_cons \\
\hline D-GIN cons. & 4 & 7 & $0.589 \pm 0.045$ & $0.734 \pm 0.043$ & dg741_cons \\
\hline D-GIN cons. & 1 & 3 & $0.561 \pm 0.047$ & $0.758 \pm 0.039$ & dg312_cons \\
\hline D-GIN cons. & 2 & 4 & $0.557 \pm 0.029$ & $0.762 \pm 0.023$ & dg422_cons \\
\hline D-GIN cons. & 4 & 3 & $0.557 \pm 0.052$ & $0.762 \pm 0.044$ & dg341_cons \\
\hline D-GIN cons. & 2 & 3 & $0.549 \pm 0.034$ & $0.769 \pm 0.026$ & dg322_cons \\
\hline D-GIN cons. & 4 & 5 & $0.590 \pm 0.059$ & $0.733 \pm 0.055$ & dg542_cons \\
\hline D-GIN cons. & 0 & 8 & $0.562 \pm 0.032$ & $0.758 \pm 0.025$ & dg801_cons \\
\hline D-GIN cons. & 0 & 4 & $0.563 \pm 0.031$ & $0.757 \pm 0.027$ & dg402_cons \\
\hline D-GIN cons. & 1 & 7 & $0.566 \pm 0.055$ & $0.754 \pm 0.044$ & dg712_cons \\
\hline D-GIN cons. & 1 & 4 & $0.567 \pm 0.051$ & $0.754 \pm 0.041$ & dg411_cons \\
\hline D-GIN cons. & 0 & 3 & $0.562 \pm 0.033$ & $0.758 \pm 0.024$ & dg302_cons \\
\hline D-GIN cons. & 4 & 8 & $0.575 \pm 0.052$ & $0.746 \pm 0.053$ & dg841_cons \\
\hline D-GIN cons. & 2 & 8 & $0.568 \pm 0.030$ & $0.753 \pm 0.026$ & dg821_cons \\
\hline D-GIN cons. & 2 & 7 & $0.580 \pm 0.027$ & $0.742 \pm 0.023$ & dg721_cons \\
\hline D-GIN cons. & 1 & 8 & $0.580 \pm 0.053$ & $0.742 \pm 0.047$ & dg811_cons \\
\hline D-GIN cons. & 4 & 4 & $0.577 \pm 0.052$ & $0.744 \pm 0.041$ & dg442_cons \\
\hline D-GIN cons. & 4 & 6 & $0.568 \pm 0.054$ & $0.751 \pm 0.051$ & dg642_cons \\
\hline D-GIN cons. & 0 & 6 & $0.569 \pm 0.029$ & $0.751 \pm 0.024$ & dg602_cons \\
\hline D-GIN cons. & 2 & 6 & $0.571 \pm 0.031$ & $0.750 \pm 0.024$ & dg622_cons \\
\hline
\end{tabular}


Table 15: Shows the logD RMSE and $r^{2}$ results for the D-MPNN model type used during this survey. The last column consist of the unique identify. Training strategy 0 represents a training strategy combining $\log \mathrm{D}, \log \mathrm{S}$ and $\log \mathrm{P}, 1$ represents a strategy combining $\log \mathrm{D}$ and $\log \mathrm{P}, 2$ stands for a combination of $\log \mathrm{D}$ and $\log \mathrm{S}, 3$ represents a strategy using $\log \mathrm{S}$ and $\log \mathrm{P}, 4$ represents a strategy using $\log \mathrm{D}, 5$ represents a strategy using $\log \mathrm{S}$ and 6 represents a strategy using $\log \mathrm{P}$.

\begin{tabular}{|c|c|c|c|c|c|}
\hline $\begin{array}{l}\text { model } \\
\text { type }\end{array}$ & $\begin{array}{l}\text { training } \\
\text { strategy }\end{array}$ & $\begin{array}{l}\text { featurization } \\
\text { strategy }\end{array}$ & $\begin{array}{l}\log \mathrm{D} \\
\mathrm{RMSE}\end{array}$ & $r^{2}$ & $\begin{array}{l}\text { unique } \\
\text { ID }\end{array}$ \\
\hline D-MPNN & 1 & 7 & $0.7836 \pm 0.065$ & $0.532 \pm 0.087$ & dmp711 \\
\hline D-MPNN & 1 & 6 & $0.686 \pm 0.054$ & $0.645 \pm 0.071$ & dmp612 \\
\hline D-MPNN & 1 & 5 & $0.857 \pm 0.060$ & $0.442 \pm 0.089$ & dmp511 \\
\hline D-MPNN & 2 & 3 & $0.759 \pm 0.037$ & $0.563 \pm 0.039$ & dmp322 \\
\hline D-MPNN & 1 & 4 & $0.692 \pm 0.069$ & $0.634 \pm 0.080$ & dmp411 \\
\hline D-MPNN & 2 & 5 & $0.911 \pm 0.029$ & $0.371 \pm 0.035$ & dmp521 \\
\hline D-MPNN & 2 & 6 & $0.721 \pm 0.029$ & $0.606 \pm 0.037$ & dmp621 \\
\hline D-MPNN & 0 & 4 & $0.721 \pm 0.036$ & $0.608 \pm 0.034$ & dmp401 \\
\hline D-MPNN & 0 & 8 & $0.712 \pm 0.039$ & $0.613 \pm 0.037$ & dmp802 \\
\hline D-MPNN & 4 & 8 & $0.713 \pm 0.043$ & $0.614 \pm 0.057$ & dmp842 \\
\hline D-MPNN & 1 & 8 & $0.724 \pm 0.044$ & $0.607 \pm 0.062$ & dmp811 \\
\hline D-MPNN & 4 & 4 & $0.719 \pm 0.057$ & $0.614 \pm 0.067$ & dmp441 \\
\hline D-MPNN & 4 & 6 & $0.719 \pm 0.056$ & $0.610 \pm 0.076$ & dmp642 \\
\hline D-MPNN & 4 & 3 & $0.731 \pm 0.044$ & $0.594 \pm 0.062$ & dmp342 \\
\hline D-MPNN & 0 & 6 & $0.724 \pm 0.030$ & $0.601 \pm 0.040$ & dmp602 \\
\hline D-MPNN & 1 & 3 & $0.703 \pm 0.084$ & $0.625 \pm 0.069$ & dmp311 \\
\hline D-MPNN & 0 & 3 & $0.719 \pm 0.040$ & $0.618 \pm 0.034$ & dmp302 \\
\hline D-MPNN & 2 & 8 & $0.788 \pm 0.027$ & $0.532 \pm 0.033$ & dmp822 \\
\hline D-MPNN & 2 & 4 & $0.728 \pm 0.041$ & $0.600 \pm 0.039$ & $\mathrm{dmp} 422$ \\
\hline D-MPNN & 2 & 7 & $0.823 \pm 0.027$ & $0.493 \pm 0.039$ & dmp721 \\
\hline D-MPNN & 4 & 7 & $0.804 \pm 0.058$ & $0.517 \pm 0.089$ & dmp742 \\
\hline D-MPNN & 4 & 5 & $0.881 \pm 0.051$ & $0.417 \pm 0.077$ & dmp542 \\
\hline D-MPNN & 0 & 7 & $0.812 \pm 0.035$ & $0.512 \pm 0.037$ & dmp702 \\
\hline D-MPNN & 0 & 5 & $0.864 \pm 0.026$ & $0.432 \pm 0.035$ & dmp502 \\
\hline D-MPNN cons. & 0 & 4 & $0.633 \pm 0.031$ & $0.693 \pm 0.025$ & dmp402_cons \\
\hline D-MPNN cons. & 4 & 5 & $0.699 \pm 0.047$ & $0.625 \pm 0.056$ & dmp542_cons \\
\hline D-MPNN cons. & 2 & 4 & $0.632 \pm 0.031$ & $0.694 \pm 0.028$ & dmp422_cons \\
\hline D-MPNN cons. & 2 & 6 & $0.632 \pm 0.029$ & $0.694 \pm 0.028$ & dmp621_cons \\
\hline D-MPNN cons. & 2 & 5 & $0.710 \pm 0.027$ & $0.614 \pm 0.024$ & dmp521_cons \\
\hline D-MPNN cons. & 1 & 8 & $0.632 \pm 0.050$ & $0.693 \pm 0.058$ & dmp811_cons \\
\hline D-MPNN cons. & 0 & 3 & $0.625 \pm 0.030$ & $0.701 \pm 0.026$ & dmp302_cons \\
\hline D-MPNN cons. & 4 & 4 & $0.625 \pm 0.054$ & $0.700 \pm 0.052$ & dmp441_cons \\
\hline D-MPNN cons. & 1 & 3 & $0.625 \pm 0.057$ & $0.700 \pm 0.050$ & dmp311_cons \\
\hline D-MPNN cons. & 0 & 8 & $0.624 \pm 0.029$ & $0.701 \pm 0.023$ & dmp802_cons \\
\hline D-MPNN cons. & 4 & 8 & $0.622 \pm 0.045$ & $0.703 \pm 0.050$ & dmp842_cons \\
\hline D-MPNN cons. & 1 & 6 & $0.618 \pm 0.045$ & $0.706 \pm 0.051$ & dmp612_cons \\
\hline D-MPNN cons. & 1 & 4 & $0.613 \pm 0.055$ & $0.711 \pm 0.057$ & dmp411_cons \\
\hline D-MPNN cons. & 4 & 6 & $0.634 \pm 0.055$ & $0.691 \pm 0.066$ & dmp642_cons \\
\hline D-MPNN cons. & 0 & 6 & $0.636 \pm 0.030$ & $0.690 \pm 0.029$ & dmp602_cons \\
\hline D-MPNN cons. & 2 & 3 & $0.646 \pm 0.032$ & $0.680 \pm 0.028$ & dmp322_cons \\
\hline D-MPNN cons. & 1 & 5 & $0.688 \pm 0.059$ & $0.637 \pm 0.057$ & dmp512_cons \\
\hline D-MPNN cons. & 2 & 8 & $0.652 \pm 0.030$ & $0.674 \pm 0.025$ & dmp822_cons \\
\hline D-MPNN cons. & 1 & 7 & $0.654 \pm 0.060$ & $0.671 \pm 0.069$ & dmp711_cons \\
\hline D-MPNN cons. & 4 & 7 & $0.663 \pm 0.045$ & $0.662 \pm 0.058$ & dmp742_cons \\
\hline D-MPNN cons. & 0 & 7 & $0.670 \pm 0.028$ & $0.656 \pm 0.026$ & dmp701_cons \\
\hline D-MPNN cons. & 2 & 7 & $0.674 \pm 0.026$ & $0.652 \pm 0.029$ & dmp721_cons \\
\hline D-MPNN cons. & 0 & 5 & $0.697 \pm 0.026$ & $0.628 \pm 0.024$ & dmp502_cons \\
\hline D-MPNN cons. & 4 & 3 & $0.636 \pm 0.042$ & $0.689 \pm 0.055$ & dmp342_cons \\
\hline
\end{tabular}


Table 16: Shows the $\log$ D RMSE and $r^{2}$ results for the GIN model type used during this survey. The last column consist of the unique identify. Training strategy 0 represents a training strategy combining $\log \mathrm{D}, \log \mathrm{S}$ and $\log \mathrm{P}, 1$ represents a strategy combining $\log \mathrm{D}$ and $\log \mathrm{P}, 2$ stands for a combination of $\log \mathrm{D}$ and $\log \mathrm{S}, 3$ represents a strategy using $\log \mathrm{S}$ and $\log \mathrm{P}, 4$ represents a strategy using $\log \mathrm{D}, 5$ represents a strategy using $\log \mathrm{S}$ and 6 represents a strategy using $\log \mathrm{P}$.

\begin{tabular}{|c|c|c|c|c|c|}
\hline $\begin{array}{l}\text { model } \\
\text { type }\end{array}$ & $\begin{array}{l}\text { training } \\
\text { strategy }\end{array}$ & $\begin{array}{l}\text { featurization } \\
\text { strategy }\end{array}$ & $\begin{array}{l}\log \mathrm{D} \\
\text { RMSE }\end{array}$ & $r^{2}$ & $\begin{array}{l}\text { unique } \\
\text { ID }\end{array}$ \\
\hline GIN & 2 & 5 & $0.860 \pm 0.035$ & $0.440 \pm 0.047$ & g522 \\
\hline GIN & 2 & 6 & $0.896 \pm 0.031$ & $0.387 \pm 0.039$ & g621 \\
\hline GIN & 0 & 4 & $0.791 \pm 0.051$ & $0.539 \pm 0.050$ & g401 \\
\hline GIN & 0 & 8 & $0.789 \pm 0.031$ & $0.526 \pm 0.036$ & g802 \\
\hline GIN & 2 & 8 & $0.814 \pm 0.036$ & $0.496 \pm 0.040$ & g822 \\
\hline GIN & 1 & 8 & $0.786 \pm 0.068$ & $0.538 \pm 0.081$ & g812 \\
\hline GIN & 1 & 6 & $0.899 \pm 0.061$ & $0.384 \pm 0.104$ & g612 \\
\hline GIN & 4 & 8 & $0.734 \pm 0.058$ & $0.589 \pm 0.079$ & g842 \\
\hline GIN & 4 & 5 & $0.856 \pm 0.073$ & $0.442 \pm 0.112$ & g542 \\
\hline GIN & 0 & 5 & $0.892 \pm 0.035$ & $0.400 \pm 0.047$ & g502 \\
\hline GIN & 4 & 3 & $0.741 \pm 0.063$ & $0.584 \pm 0.080$ & g342 \\
\hline GIN & 1 & 7 & $0.756 \pm 0.060$ & $0.567 \pm 0.057$ & g711 \\
\hline GIN & 1 & 4 & $0.761 \pm 0.076$ & $0.561 \pm 0.071$ & g412 \\
\hline GIN & 4 & 4 & $0.743 \pm 0.066$ & $0.581 \pm 0.061$ & g441 \\
\hline GIN & 4 & 7 & $0.747 \pm 0.082$ & $0.576 \pm 0.087$ & g742 \\
\hline GIN & 2 & 7 & $0.751 \pm 0.041$ & $0.581 \pm 0.040$ & g722 \\
\hline GIN & 2 & 3 & $0.781 \pm 0.035$ & $0.557 \pm 0.041$ & g321 \\
\hline GIN & 2 & 4 & $0.766 \pm 0.042$ & $0.557 \pm 0.044$ & g421 \\
\hline GIN & 0 & 7 & $0.765 \pm 0.040$ & $0.558 \pm 0.041$ & g702 \\
\hline GIN & 0 & 3 & $0.756 \pm 0.030$ & $0.570 \pm 0.033$ & g302 \\
\hline GIN & 1 & 3 & $0.742 \pm 0.065$ & $0.580 \pm 0.062$ & g311 \\
\hline GIN & 4 & 6 & $0.860 \pm 0.063$ & $0.437 \pm 0.085$ & g642 \\
\hline GIN & 0 & 6 & $0.900 \pm 0.033$ & $0.381 \pm 0.041$ & g601 \\
\hline GIN & 1 & 5 & $0.911 \pm 0.056$ & $0.371 \pm 0.062$ & g512 \\
\hline GINcons. & 0 & 6 & $0.715 \pm 0.020$ & $0.609 \pm 0.025$ & g602_cons \\
\hline GINcons. & 4 & 3 & $0.627 \pm 0.054$ & $0.698 \pm 0.059$ & g342_cons \\
\hline GINcons. & 4 & 8 & $0.632 \pm 0.050$ & $0.693 \pm 0.057$ & g842_cons \\
\hline GINcons. & 4 & 7 & $0.633 \pm 0.061$ & $0.692 \pm 0.056$ & g742_cons \\
\hline GINcons. & 4 & 4 & $0.637 \pm 0.058$ & $0.688 \pm 0.060$ & g441_cons \\
\hline GINcons. & 1 & 3 & $0.640 \pm 0.056$ & $0.685 \pm 0.051$ & g311_cons \\
\hline GINcons. & 2 & 7 & $0.642 \pm 0.033$ & $0.685 \pm 0.028$ & g722_cons \\
\hline GINcons. & 1 & 7 & $0.644 \pm 0.047$ & $0.682 \pm 0.053$ & g711_cons \\
\hline GINcons. & 2 & 4 & $0.645 \pm 0.034$ & $0.681 \pm 0.031$ & g421_cons \\
\hline GINcons. & 0 & 3 & $0.647 \pm 0.029$ & $0.679 \pm 0.025$ & g302_cons \\
\hline GINcons. & 1 & 4 & $0.648 \pm 0.059$ & $0.678 \pm 0.056$ & g412_cons \\
\hline GINcons. & 0 & 4 & $0.652 \pm 0.033$ & $0.674 \pm 0.030$ & g401_cons \\
\hline GINcons. & 0 & 7 & $0.654 \pm 0.029$ & $0.673 \pm 0.024$ & g701_cons \\
\hline GINcons. & 2 & 3 & $0.656 \pm 0.028$ & $0.670 \pm 0.030$ & g321_cons \\
\hline GINcons. & 1 & 8 & $0.657 \pm 0.055$ & $0.669 \pm 0.068$ & g812_cons \\
\hline GINcons. & 0 & 8 & $0.661 \pm 0.034$ & $0.665 \pm 0.031$ & g801_cons \\
\hline GINcons. & 2 & 8 & $0.673 \pm 0.032$ & $0.653 \pm 0.029$ & g822_cons \\
\hline GINcons. & 4 & 5 & $0.689 \pm 0.056$ & $0.636 \pm 0.064$ & g542_cons \\
\hline GINcons. & 4 & 6 & $0.692 \pm 0.052$ & $0.633 \pm 0.057$ & g642_cons \\
\hline GINcons. & 1 & 6 & $0.707 \pm 0.057$ & $0.616 \pm 0.061$ & g612_cons \\
\hline GINcons. & 2 & 6 & $0.707 \pm 0.025$ & $0.617 \pm 0.025$ & g622_cons \\
\hline GINcons. & 0 & 5 & $0.712 \pm 0.033$ & $0.612 \pm 0.030$ & g502_cons \\
\hline GINcons. & 1 & 5 & $0.719 \pm 0.046$ & $0.603 \pm 0.052$ & g512_cons \\
\hline GINcons. & 2 & 5 & $0.692 \pm 0.026$ & $0.634 \pm 0.022$ & g522_cons \\
\hline
\end{tabular}


Table 17: Shows the logD RMSE and $r^{2}$ results for the non-GNN model types used during this survey. The last column consist of the unique identify. Training strategy 0 represents a training strategy combining $\log \mathrm{D}, \log \mathrm{S}$ and $\log \mathrm{P}, 1$ represents a strategy combining $\log \mathrm{D}$ and $\log \mathrm{P}, 2$ stands for a combination of $\log \mathrm{D}$ and $\log \mathrm{S}, 3$ represents a strategy using $\log \mathrm{S}$ and $\log \mathrm{P}, 4$ represents a strategy using $\log \mathrm{D}, 5$ represents a strategy using $\log \mathrm{S}$ and 6 represents a strategy using $\log \mathrm{P}$.

\begin{tabular}{llllll}
\hline $\begin{array}{l}\text { model } \\
\text { type }\end{array}$ & $\begin{array}{l}\text { training } \\
\text { strategy }\end{array}$ & $\begin{array}{l}\text { featurization } \\
\text { strategy }\end{array}$ & $\begin{array}{l}\log \mathrm{R} \\
\text { RMSE }\end{array}$ & $r^{2}$ & unique \\
\hline KNN & $\log \mathrm{D}$ & 10 & $0.979 \pm 0.072$ & $0.261 \pm 0.094$ & KNN10 \\
KNN & $\log \mathrm{D}$ & 16 & $0.970 \pm 0.056$ & $0.275 \pm 0.097$ & KNN16 \\
KNN & $\log \mathrm{D}$ & 12 & $0.959 \pm 0.066$ & $0.293 \pm 0.080$ & KNN12 \\
KNN & $\log \mathrm{D}$ & 11 & $0.993 \pm 0.072$ & $0.240 \pm 0.096$ & KNN11 \\
KNN & $\log \mathrm{D}$ & 13 & $0.909 \pm 0.067$ & $0.363 \pm 0.069$ & KNN13 \\
KNN & $\log \mathrm{D}$ & 15 & $1.003 \pm 0.057$ & $0.226 \pm 0.096$ & KNN15 \\
KNN & $\log \mathrm{D}$ & 14 & $0.996 \pm 0.065$ & $0.236 \pm 0.110$ & KNN14 \\
KNN & $\log \mathrm{D}$ & 17 & $0.801 \pm 0.060$ & $0.506 \pm 0.058$ & KNN17 \\
RF & $\log \mathrm{D}$ & 17 & $0.708 \pm 0.060$ & $0.614 \pm 0.055$ & rf17 \\
RF & $\log \mathrm{D}$ & 15 & $0.699 \pm 0.062$ & $0.623 \pm 0.059$ & rf15 \\
RF & $\log \mathrm{D}$ & 16 & $0.703 \pm 0.061$ & $0.620 \pm 0.057$ & rf16 \\
RF & $\log \mathrm{D}$ & 11 & $0.859 \pm 0.062$ & $0.433 \pm 0.062$ & rf11 \\
RF & $\log \mathrm{D}$ & 14 & $0.706 \pm 0.062$ & $0.616 \pm 0.058$ & rf14 \\
RF & $\log \mathrm{D}$ & 10 & $0.890 \pm 0.060$ & $0.390 \pm 0.062$ & rf10 \\
RF & $\log \mathrm{D}$ & 13 & $0.813 \pm 0.060$ & $0.491 \pm 0.059$ & rf13 \\
RF & $\log \mathrm{D}$ & 12 & $0.863 \pm 0.068$ & $0.427 \pm 0.068$ & rf12 \\
SVM & $\log \mathrm{D}$ & 12 & $0.782 \pm 0.061$ & $0.529 \pm 0.055$ & svm12 \\
SVM & $\log \mathrm{D}$ & 16 & $0.707 \pm 0.056$ & $0.615 \pm 0.047$ & svm16 \\
SVM & $\log \mathrm{D}$ & 10 & $0.810 \pm 0.062$ & $0.495 \pm 0.057$ & svm10 \\
SVM & $\log \mathrm{D}$ & 15 & $0.698 \pm 0.055$ & $0.625 \pm 0.045$ & svm15 \\
SVM & $\log \mathrm{D}$ & 14 & $0.674 \pm 0.054$ & $0.650 \pm 0.043$ & svm14 \\
SVM & $\log \mathrm{D}$ & 17 & $0.639 \pm 0.051$ & $0.686 \pm 0.046$ & svm17 \\
SVM & $\log \mathrm{D}$ & 11 & $0.793 \pm 0.060$ & $0.516 \pm 0.060$ & svm11 \\
SVM & $\log \mathrm{D}$ & 13 & $0.814 \pm 0.059$ & $0.490 \pm 0.055$ & svm13 \\
\hline
\end{tabular}


Table 18: Shows the logS RMSE and $r^{2}$ results for the D-GIN model type used during this survey. The last column consist of the unique identify. Training strategy 0 represents a training strategy combining $\log \mathrm{D}, \log \mathrm{S}$ and $\log \mathrm{P}, 1$ represents a strategy combining $\log \mathrm{D}$ and $\log \mathrm{P}, 2$ stands for a combination of $\log \mathrm{D}$ and $\log \mathrm{S}, 3$ represents a strategy using $\log \mathrm{S}$ and $\log \mathrm{P}, 4$ represents a strategy using $\log \mathrm{D}, 5$ represents a strategy using $\log \mathrm{S}$ and 6 represents a strategy using $\log \mathrm{P}$.

\begin{tabular}{|c|c|c|c|c|c|}
\hline $\begin{array}{l}\text { model } \\
\text { type }\end{array}$ & $\begin{array}{l}\text { training } \\
\text { strategy }\end{array}$ & $\begin{array}{l}\text { featurization } \\
\text { strategy }\end{array}$ & $\begin{array}{l}\log S \\
\text { RMSE }\end{array}$ & $r^{2}$ & $\begin{array}{l}\text { unique } \\
\text { ID }\end{array}$ \\
\hline D-GIN & 5 & 7 & $0.897 \pm 0.041$ & $0.757 \pm 0.025$ & $\operatorname{dg} 752$ \\
\hline D-GIN & 0 & 7 & $0.866 \pm 0.034$ & $0.771 \pm 0.016$ & dg701 \\
\hline D-GIN & 5 & 5 & $0.969 \pm 0.048$ & $0.717 \pm 0.030$ & $\operatorname{dg} 552$ \\
\hline D-GIN & 0 & 5 & $0.988 \pm 0.042$ & $0.705 \pm 0.021$ & dg502 \\
\hline D-GIN & 2 & 5 & $1.010 \pm 0.040$ & $0.694 \pm 0.020$ & dg522 \\
\hline D-GIN & 0 & 8 & $0.795 \pm 0.038$ & $0.807 \pm 0.019$ & dg801 \\
\hline D-GIN & 0 & 4 & $0.807 \pm 0.032$ & $0.803 \pm 0.016$ & $\operatorname{dg} 401$ \\
\hline D-GIN & 3 & 8 & $0.813 \pm 0.044$ & $0.813 \pm 0.021$ & dg832 \\
\hline D-GIN & 5 & 6 & $0.818 \pm 0.044$ & $0.798 \pm 0.025$ & $\operatorname{dg} 651$ \\
\hline D-GIN & 5 & 3 & $0.848 \pm 0.047$ & $0.783 \pm 0.023$ & dg352 \\
\hline D-GIN & 0 & 6 & $0.821 \pm 0.042$ & $0.796 \pm 0.020$ & $\operatorname{dg} 601$ \\
\hline D-GIN & 2 & 7 & $0.906 \pm 0.045$ & $0.755 \pm 0.017$ & dg721 \\
\hline D-GIN & 2 & 4 & $0.822 \pm 0.028$ & $0.794 \pm 0.014$ & $\operatorname{dg} 422$ \\
\hline D-GIN & 3 & 6 & $0.827 \pm 0.063$ & $0.794 \pm 0.036$ & $\operatorname{dg} 632$ \\
\hline D-GIN & 5 & 8 & $0.854 \pm 0.056$ & $0.781 \pm 0.027$ & dg852 \\
\hline D-GIN & 3 & 4 & $0.829 \pm 0.044$ & $0.791 \pm 0.025$ & $\operatorname{dg} 432$ \\
\hline D-GIN & 3 & 3 & $0.831 \pm 0.050$ & $0.790 \pm 0.026$ & $\operatorname{dg} 332$ \\
\hline D-GIN & 0 & 3 & $0.832 \pm 0.039$ & $0.798 \pm 0.016$ & dg302 \\
\hline D-GIN & 2 & 3 & $0.833 \pm 0.037$ & $0.789 \pm 0.016$ & dg321 \\
\hline D-GIN & 5 & 4 & $0.852 \pm 0.050$ & $0.789 \pm 0.026$ & $\operatorname{dg} 451$ \\
\hline D-GIN & 3 & 7 & $0.851 \pm 0.049$ & $0.782 \pm 0.027$ & $\operatorname{dg} 732$ \\
\hline D-GIN & 2 & 6 & $0.837 \pm 0.042$ & $0.788 \pm 0.020$ & $\operatorname{dg} 622$ \\
\hline D-GIN & 3 & 5 & $1.061 \pm 0.061$ & $0.662 \pm 0.034$ & dg532 \\
\hline D-GIN cons. & 2 & 5 & $0.794 \pm 0.039$ & $0.807 \pm 0.014$ & dg522_cons \\
\hline D-GIN cons. & 0 & 5 & $0.779 \pm 0.040$ & $0.814 \pm 0.014$ & dg502_cons \\
\hline D-GIN cons. & 5 & 5 & $0.778 \pm 0.049$ & $0.816 \pm 0.020$ & dg552_cons \\
\hline D-GIN cons. & 3 & 5 & $0.820 \pm 0.054$ & $0.795 \pm 0.024$ & dg532_cons \\
\hline D-GIN cons. & 0 & 8 & $0.705 \pm 0.039$ & $0.848 \pm 0.014$ & dg801_cons \\
\hline D-GIN cons. & 2 & 7 & $0.757 \pm 0.044$ & $0.825 \pm 0.015$ & dg721_cons \\
\hline D-GIN cons. & 2 & 8 & $0.734 \pm 0.033$ & $0.835 \pm 0.012$ & dg822_cons \\
\hline D-GIN cons. & 3 & 7 & $0.733 \pm 0.046$ & $0.836 \pm 0.021$ & dg732_cons \\
\hline D-GIN cons. & 0 & 3 & $0.733 \pm 0.040$ & $0.836 \pm 0.015$ & dg301_cons \\
\hline D-GIN cons. & 0 & 7 & $0.731 \pm 0.035$ & $0.836 \pm 0.010$ & dg701_cons \\
\hline D-GIN cons. & 5 & 3 & $0.730 \pm 0.047$ & $0.838 \pm 0.019$ & dg352_cons \\
\hline D-GIN cons. & 3 & 3 & $0.727 \pm 0.048$ & $0.839 \pm 0.021$ & dg332_cons \\
\hline D-GIN cons. & 5 & 4 & $0.739 \pm 0.052$ & $0.834 \pm 0.023$ & dg451_cons \\
\hline D-GIN cons. & 5 & 7 & $0.748 \pm 0.045$ & $0.830 \pm 0.018$ & dg752_cons \\
\hline D-GIN cons. & 2 & 3 & $0.725 \pm 0.041$ & $0.839 \pm 0.014$ & dg321_cons \\
\hline D-GIN cons. & 3 & 8 & $0.724 \pm 0.046$ & $0.840 \pm 0.018$ & dg832_cons \\
\hline D-GIN cons. & 3 & 4 & $0.722 \pm 0.047$ & $0.841 \pm 0.020$ & dg432_cons \\
\hline D-GIN cons. & 2 & 4 & $0.718 \pm 0.031$ & $0.842 \pm 0.011$ & dg422_cons \\
\hline D-GIN cons. & 3 & 6 & $0.718 \pm 0.047$ & $0.843 \pm 0.021$ & dg631_cons \\
\hline D-GIN cons. & 2 & 6 & $0.716 \pm 0.038$ & $0.843 \pm 0.014$ & dg622_cons \\
\hline D-GIN cons. & 0 & 4 & $0.715 \pm 0.035$ & $0.843 \pm 0.011$ & dg401_cons \\
\hline D-GIN cons. & 5 & 6 & $0.711 \pm 0.046$ & $0.846 \pm 0.021$ & dg651_cons \\
\hline D-GIN cons. & 0 & 6 & $0.724 \pm 0.038$ & $0.839 \pm 0.016$ & dg601_cons \\
\hline D-GIN cons. & 5 & 8 & $0.735 \pm 0.053$ & $0.835 \pm 0.021$ & dg852_cons \\
\hline
\end{tabular}


Table 19: Shows the logS RMSE and $r^{2}$ results for the D-MPNN model type used during this survey. The last column consist of the unique identify. Training strategy 0 represents a training strategy combining $\log \mathrm{D}, \log \mathrm{S}$ and $\log \mathrm{P}, 1$ represents a strategy combining $\log \mathrm{D}$ and $\log \mathrm{P}, 2$ stands for a combination of $\log \mathrm{D}$ and $\log \mathrm{S}, 3$ represents a strategy using $\log \mathrm{S}$ and $\log \mathrm{P}, 4$ represents a strategy using $\log \mathrm{D}, 5$ represents a strategy using $\log \mathrm{S}$ and 6 represents a strategy using $\log$.

\begin{tabular}{|c|c|c|c|c|c|}
\hline $\begin{array}{l}\text { model } \\
\text { type }\end{array}$ & $\begin{array}{l}\text { training } \\
\text { strategy }\end{array}$ & $\begin{array}{l}\text { featurization } \\
\text { strategy }\end{array}$ & $\begin{array}{l}\log S \\
\text { RMSE }\end{array}$ & $r^{2}$ & $\begin{array}{l}\text { unique } \\
\text { ID }\end{array}$ \\
\hline D-MPNN & 3 & 8 & $0.913 \pm 0.052$ & $0.756 \pm 0.026$ & dmp832 \\
\hline D-MPNN & 2 & 6 & $0.857 \pm 0.035$ & $0.782 \pm 0.015$ & dmp621 \\
\hline D-MPNN & 2 & 3 & $0.865 \pm 0.038$ & $0.784 \pm 0.016$ & dmp322 \\
\hline D-MPNN & 0 & 5 & $0.962 \pm 0.038$ & $0.718 \pm 0.018$ & dmp501 \\
\hline D-MPNN & 0 & 8 & $0.907 \pm 0.049$ & $0.757 \pm 0.021$ & dmp802 \\
\hline D-MPNN & 5 & 8 & $0.921 \pm 0.046$ & $0.751 \pm 0.025$ & dmp851 \\
\hline D-MPNN & 2 & 8 & $0.906 \pm 0.041$ & $0.763 \pm 0.020$ & dmp821 \\
\hline D-MPNN & 0 & 7 & $0.906 \pm 0.049$ & $0.764 \pm 0.027$ & dmp701 \\
\hline D-MPNN & 2 & 7 & $0.902 \pm 0.040$ & $0.772 \pm 0.021$ & dmp722 \\
\hline D-MPNN & 3 & 5 & $0.938 \pm 0.044$ & $0.733 \pm 0.029$ & dmp532 \\
\hline D-MPNN & 5 & 5 & $0.945 \pm 0.041$ & $0.734 \pm 0.030$ & dmp552 \\
\hline D-MPNN & 3 & 4 & $0.896 \pm 0.062$ & $0.782 \pm 0.028$ & $\mathrm{dmp} 432$ \\
\hline D-MPNN & 0 & 4 & $0.893 \pm 0.040$ & $0.762 \pm 0.022$ & $\mathrm{dmp} 401$ \\
\hline D-MPNN & 3 & 3 & $0.893 \pm 0.054$ & $0.775 \pm 0.026$ & dmp332 \\
\hline D-MPNN & 5 & 6 & $0.879 \pm 0.046$ & $0.777 \pm 0.021$ & dmp651 \\
\hline D-MPNN & 5 & 3 & $0.878 \pm 0.053$ & $0.772 \pm 0.027$ & dmp351 \\
\hline D-MPNN & 0 & 6 & $0.877 \pm 0.039$ & $0.780 \pm 0.021$ & dmp601 \\
\hline D-MPNN & 0 & 3 & $0.874 \pm 0.046$ & $0.779 \pm 0.024$ & dmp302 \\
\hline D-MPNN & 2 & 5 & $0.961 \pm 0.036$ & $0.721 \pm 0.021$ & dmp521 \\
\hline D-MPNN & 5 & 4 & $0.866 \pm 0.054$ & $0.778 \pm 0.022$ & $\mathrm{dmp} 452$ \\
\hline D-MPNN & 3 & 7 & $0.865 \pm 0.049$ & $0.773 \pm 0.027$ & dmp731 \\
\hline D-MPNN & 2 & 4 & $0.863 \pm 0.046$ & $0.800 \pm 0.020$ & $\mathrm{dmp} 422$ \\
\hline D-MPNN & 5 & 7 & $0.897 \pm 0.050$ & $0.763 \pm 0.024$ & dmp751 \\
\hline D-MPNN & 3 & 6 & $0.861 \pm 0.047$ & $0.780 \pm 0.024$ & dmp632 \\
\hline D-MPNN cons. & 5 & 4 & $0.748 \pm 0.055$ & $0.830 \pm 0.020$ & dmp452_cons \\
\hline D-MPNN cons. & 0 & 4 & $0.768 \pm 0.040$ & $0.819 \pm 0.015$ & dmp401_cons \\
\hline D-MPNN cons. & 2 & 8 & $0.769 \pm 0.039$ & $0.819 \pm 0.014$ & dmp821_cons \\
\hline D-MPNN cons. & 3 & 8 & $0.762 \pm 0.052$ & $0.823 \pm 0.022$ & dmp832_cons \\
\hline D-MPNN cons. & 5 & 6 & $0.749 \pm 0.053$ & $0.829 \pm 0.021$ & dmp652_cons \\
\hline D-MPNN cons. & 0 & 8 & $0.770 \pm 0.044$ & $0.818 \pm 0.016$ & dmp802_cons \\
\hline D-MPNN cons. & 3 & 3 & $0.762 \pm 0.052$ & $0.823 \pm 0.021$ & dmp332_cons \\
\hline D-MPNN cons. & 0 & 7 & $0.771 \pm 0.044$ & $0.818 \pm 0.016$ & dmp701_cons \\
\hline D-MPNN cons. & 2 & 7 & $0.771 \pm 0.040$ & $0.818 \pm 0.016$ & dmp722_cons \\
\hline D-MPNN cons. & 5 & 7 & $0.761 \pm 0.051$ & $0.824 \pm 0.021$ & dmp751_cons \\
\hline D-MPNN cons. & 5 & 8 & $0.774 \pm 0.048$ & $0.817 \pm 0.022$ & dmp851_cons \\
\hline D-MPNN cons. & 3 & 5 & $0.777 \pm 0.048$ & $0.816 \pm 0.021$ & dmp532_cons \\
\hline D-MPNN cons. & 5 & 5 & $0.779 \pm 0.045$ & $0.815 \pm 0.021$ & dmp552_cons \\
\hline D-MPNN cons. & 3 & 4 & $0.765 \pm 0.056$ & $0.822 \pm 0.021$ & dmp431_cons \\
\hline D-MPNN cons. & 2 & 5 & $0.784 \pm 0.038$ & $0.811 \pm 0.016$ & dmp521_cons \\
\hline D-MPNN cons. & 0 & 3 & $0.760 \pm 0.042$ & $0.823 \pm 0.016$ & dmp302_cons \\
\hline D-MPNN cons. & 0 & 6 & $0.759 \pm 0.039$ & $0.823 \pm 0.014$ & dmp601_cons \\
\hline D-MPNN cons. & 2 & 3 & $0.756 \pm 0.039$ & $0.825 \pm 0.014$ & dmp322_cons \\
\hline D-MPNN cons. & 2 & 6 & $0.744 \pm 0.038$ & $0.831 \pm 0.013$ & dmp621_cons \\
\hline D-MPNN cons. & 5 & 3 & $0.752 \pm 0.055$ & $0.828 \pm 0.021$ & dmp351_cons \\
\hline D-MPNN cons. & 3 & 7 & $0.744 \pm 0.053$ & $0.831 \pm 0.021$ & dmp731_cons \\
\hline D-MPNN cons. & 2 & 4 & $0.750 \pm 0.044$ & $0.828 \pm 0.016$ & dmp422_cons \\
\hline D-MPNN cons. & 3 & 6 & $0.748 \pm 0.055$ & $0.830 \pm 0.021$ & dmp632_cons \\
\hline D-MPNN cons. & 0 & 5 & $0.785 \pm 0.040$ & $0.811 \pm 0.015$ & dmp501_cons \\
\hline
\end{tabular}


Table 20: Shows the logS RMSE and $r^{2}$ results for the GIN model type used during this survey. The last column consist of the unique identify. Training strategy 0 represents a training strategy combining $\log \mathrm{D}, \log \mathrm{S}$ and $\log \mathrm{P}, 1$ represents a strategy combining $\log \mathrm{D}$ and $\log \mathrm{P}, 2$ stands for a combination of $\log \mathrm{D}$ and $\log \mathrm{S}, 3$ represents a strategy using $\log \mathrm{S}$ and $\log \mathrm{P}, 4$ represents a strategy using $\log \mathrm{D}, 5$ represents a strategy using $\log \mathrm{S}$ and 6 represents a strategy using $\log \mathrm{P}$.

\begin{tabular}{|c|c|c|c|c|c|}
\hline $\begin{array}{l}\text { model } \\
\text { type }\end{array}$ & $\begin{array}{l}\text { training } \\
\text { strategy }\end{array}$ & $\begin{array}{l}\text { featurization } \\
\text { strategy }\end{array}$ & $\begin{array}{l}\log S \\
\text { RMSE }\end{array}$ & $r^{2}$ & $\begin{array}{l}\text { unique } \\
\text { ID }\end{array}$ \\
\hline GIN & 2 & 4 & $1.126 \pm 0.047$ & $0.612 \pm 0.029$ & g421 \\
\hline GIN & 0 & 3 & $1.122 \pm 0.049$ & $0.615 \pm 0.028$ & g301 \\
\hline GIN & 0 & 4 & $1.116 \pm 0.053$ & $0.624 \pm 0.037$ & g402 \\
\hline GIN & 0 & 7 & $1.089 \pm 0.044$ & $0.650 \pm 0.031$ & g701 \\
\hline GIN & 3 & 7 & $1.088 \pm 0.066$ & $0.643 \pm 0.036$ & g731 \\
\hline GIN & 2 & 7 & $1.127 \pm 0.049$ & $0.613 \pm 0.028$ & g721 \\
\hline GIN & 3 & 4 & $1.147 \pm 0.084$ & $0.602 \pm 0.052$ & g431 \\
\hline GIN & 5 & 7 & $1.145 \pm 0.060$ & $0.602 \pm 0.038$ & g752 \\
\hline GIN & 5 & 6 & $1.400 \pm 0.064$ & $0.407 \pm 0.045$ & g652 \\
\hline GIN & 5 & 5 & $1.362 \pm 0.061$ & $0.437 \pm 0.043$ & g552 \\
\hline GIN & 2 & 6 & $1.359 \pm 0.045$ & $0.441 \pm 0.036$ & g621 \\
\hline GIN & 2 & 5 & $1.345 \pm 0.044$ & $0.447 \pm 0.030$ & g522 \\
\hline GIN & 3 & 6 & $1.334 \pm 0.058$ & $0.463 \pm 0.047$ & g631 \\
\hline GIN & 0 & 5 & $1.326 \pm 0.049$ & $0.474 \pm 0.031$ & g502 \\
\hline GIN & 0 & 8 & $1.136 \pm 0.058$ & $0.605 \pm 0.034$ & g801 \\
\hline GIN & 3 & 5 & $1.326 \pm 0.066$ & $0.472 \pm 0.049$ & g532 \\
\hline GIN & 5 & 8 & $1.217 \pm 0.062$ & $0.554 \pm 0.040$ & g851 \\
\hline GIN & 5 & 3 & $1.178 \pm 0.070$ & $0.579 \pm 0.042$ & g352 \\
\hline GIN & 2 & 8 & $1.173 \pm 0.050$ & $0.580 \pm 0.027$ & g821 \\
\hline GIN & 3 & 8 & $1.166 \pm 0.059$ & $0.603 \pm 0.036$ & g832 \\
\hline GIN & 5 & 4 & $1.161 \pm 0.089$ & $0.598 \pm 0.057$ & g452 \\
\hline GIN & 2 & 3 & $1.151 \pm 0.047$ & $0.595 \pm 0.025$ & g321 \\
\hline GIN & 3 & 3 & $1.148 \pm 0.064$ & $0.610 \pm 0.036$ & g331 \\
\hline GIN & 0 & 6 & $1.314 \pm 0.053$ & $0.474 \pm 0.034$ & g602 \\
\hline GINcons. & 5 & 6 & $0.969 \pm 0.057$ & $0.714 \pm 0.029$ & g652_cons \\
\hline GINcons. & 2 & 7 & $0.840 \pm 0.036$ & $0.784 \pm 0.014$ & g721_cons \\
\hline GINcons. & 5 & 5 & $0.945 \pm 0.045$ & $0.728 \pm 0.023$ & g552_cons \\
\hline GINcons. & 0 & 3 & $0.842 \pm 0.041$ & $0.783 \pm 0.016$ & g301_cons \\
\hline GINcons. & 5 & 4 & $0.861 \pm 0.065$ & $0.774 \pm 0.031$ & g452_cons \\
\hline GINcons. & 2 & 8 & $0.867 \pm 0.043$ & $0.770 \pm 0.017$ & g821_cons \\
\hline GINcons. & 5 & 3 & $0.872 \pm 0.056$ & $0.769 \pm 0.026$ & g352_cons \\
\hline GINcons. & 3 & 3 & $0.857 \pm 0.054$ & $0.776 \pm 0.024$ & g331_cons \\
\hline GINcons. & 0 & 7 & $0.825 \pm 0.038$ & $0.791 \pm 0.016$ & g701_cons \\
\hline GINcons. & 5 & 8 & $0.887 \pm 0.056$ & $0.760 \pm 0.026$ & g851_cons \\
\hline GINcons. & 2 & 3 & $0.855 \pm 0.043$ & $0.776 \pm 0.015$ & g321_cons \\
\hline GINcons. & 5 & 7 & $0.854 \pm 0.055$ & $0.778 \pm 0.026$ & g752_cons \\
\hline GINcons. & 3 & 7 & $0.828 \pm 0.052$ & $0.791 \pm 0.023$ & g731_cons \\
\hline GINcons. & 0 & 4 & $0.835 \pm 0.040$ & $0.786 \pm 0.016$ & g402_cons \\
\hline GINcons. & 3 & 4 & $0.860 \pm 0.061$ & $0.775 \pm 0.029$ & g431_cons \\
\hline GINcons. & 2 & 4 & $0.835 \pm 0.041$ & $0.786 \pm 0.014$ & g421_cons \\
\hline GINcons. & 0 & 6 & $0.926 \pm 0.045$ & $0.737 \pm 0.017$ & g602_cons \\
\hline GINcons. & 0 & 5 & $0.933 \pm 0.038$ & $0.733 \pm 0.015$ & g502_cons \\
\hline GINcons. & 3 & 5 & $0.934 \pm 0.053$ & $0.734 \pm 0.025$ & g532_cons \\
\hline GINcons. & 2 & 5 & $0.938 \pm 0.040$ & $0.730 \pm 0.015$ & g522_cons \\
\hline GINcons. & 3 & 6 & $0.940 \pm 0.048$ & $0.731 \pm 0.025$ & g631_cons \\
\hline GINcons. & 2 & 6 & $0.945 \pm 0.041$ & $0.726 \pm 0.017$ & g621_cons \\
\hline GINcons. & 0 & 8 & $0.850 \pm 0.042$ & $0.779 \pm 0.014$ & g802_cons \\
\hline GINcons. & 3 & 8 & $0.861 \pm 0.053$ & $0.774 \pm 0.018$ & g832_cons \\
\hline
\end{tabular}


Table 21: Shows the logS RMSE and $r^{2}$ results for the non-GNN model types used during this survey. The last column consist of the unique identify. Training strategy 0 represents a training strategy combining $\log \mathrm{D}, \log \mathrm{S}$ and $\log \mathrm{P}, 1$ represents a strategy combining $\log \mathrm{D}$ and $\log \mathrm{P}, 2$ stands for a combination of $\log \mathrm{D}$ and $\log \mathrm{S}, 3$ represents a strategy using $\log \mathrm{S}$ and $\log \mathrm{P}, 4$ represents a strategy using $\log \mathrm{D}, 5$ represents a strategy using $\log \mathrm{S}$ and 6 represents a strategy using $\log \mathrm{P}$.

\begin{tabular}{|c|c|c|c|c|c|}
\hline $\begin{array}{l}\text { model } \\
\text { type }\end{array}$ & $\begin{array}{l}\text { training } \\
\text { strategy }\end{array}$ & $\begin{array}{l}\text { featurization } \\
\text { strategy }\end{array}$ & $\begin{array}{l}\log S \\
\text { RMSE }\end{array}$ & $r^{2}$ & $\begin{array}{l}\text { unique } \\
\text { ID }\end{array}$ \\
\hline KNN & $\log S$ & 14 & $1.547 \pm 0.063$ & $0.268 \pm 0.054$ & KNN14 \\
\hline KNN & $\log S$ & 11 & $1.587 \pm 0.063$ & $0.230 \pm 0.054$ & KNN11 \\
\hline KNN & $\log S$ & 10 & $1.587 \pm 0.064$ & $0.229 \pm 0.056$ & KNN10 \\
\hline KNN & $\log S$ & 12 & $1.600 \pm 0.066$ & $0.217 \pm 0.055$ & KNN12 \\
\hline KNN & $\log S$ & 13 & $1.280 \pm 0.066$ & $0.499 \pm 0.036$ & KNN13 \\
\hline KNN & $\log S$ & 15 & $1.676 \pm 0.066$ & $0.140 \pm 0.068$ & KNN15 \\
\hline KNN & $\log S$ & 17 & $1.058 \pm 0.055$ & $0.658 \pm 0.032$ & KNN17 \\
\hline KNN & $\log S$ & 16 & $1.670 \pm 0.065$ & $0.147 \pm 0.061$ & KNN16 \\
\hline RF & $\log S$ & 12 & $1.239 \pm 0.056$ & $0.530 \pm 0.032$ & rf12 \\
\hline RF & $\log S$ & 14 & $0.760 \pm 0.043$ & $0.823 \pm 0.020$ & rf14 \\
\hline RF & $\log S$ & 15 & $0.764 \pm 0.044$ & $0.821 \pm 0.022$ & rf15 \\
\hline RF & $\log S$ & 13 & $1.128 \pm 0.061$ & $0.611 \pm 0.039$ & rf13 \\
\hline RF & $\log S$ & 16 & $0.765 \pm 0.045$ & $0.821 \pm 0.024$ & rf16 \\
\hline RF & $\log S$ & 17 & $0.770 \pm 0.049$ & $0.818 \pm 0.025$ & rf17 \\
\hline RF & $\log S$ & 10 & $1.284 \pm 0.055$ & $0.495 \pm 0.033$ & rf10 \\
\hline RF & $\log S$ & 11 & $1.271 \pm 0.057$ & $0.506 \pm 0.035$ & rf11 \\
\hline SVM & $\log S$ & 12 & $1.142 \pm 0.055$ & $0.601 \pm 0.035$ & svm12 \\
\hline SVM & $\log S$ & 11 & $1.149 \pm 0.057$ & $0.596 \pm 0.035$ & svm11 \\
\hline SVM & $\log S$ & 16 & $0.966 \pm 0.049$ & $0.715 \pm 0.024$ & svm16 \\
\hline SVM & $\log S$ & 15 & $0.930 \pm 0.046$ & $0.735 \pm 0.023$ & svm15 \\
\hline SVM & $\log S$ & 13 & $1.086 \pm 0.060$ & $0.639 \pm 0.034$ & svm13 \\
\hline SVM & $\log S$ & 17 & $0.730 \pm 0.041$ & $0.837 \pm 0.020$ & svm17 \\
\hline SVM & $\log S$ & 14 & $0.891 \pm 0.046$ & $0.757 \pm 0.023$ & svm14 \\
\hline SVM & $\log S$ & 10 & $1.162 \pm 0.056$ & $0.587 \pm 0.036$ & svm10 \\
\hline
\end{tabular}


Table 22: Shows the $\log$ P RMSE and $r^{2}$ results for the D-GIN model type used during this survey. The last column consist of the unique identify. Training strategy 0 represents a training strategy combining $\log \mathrm{D}, \log \mathrm{S}$ and $\log \mathrm{P}, 1$ represents a strategy combining $\log \mathrm{D}$ and $\log \mathrm{P}, 2$ stands for a combination of $\log \mathrm{D}$ and $\log \mathrm{S}, 3$ represents a strategy using $\log \mathrm{S}$ and $\log \mathrm{P}, 4$ represents a strategy using $\log \mathrm{D}, 5$ represents a strategy using $\log \mathrm{S}$ and 6 represents a strategy using $\log \mathrm{P}$.

\begin{tabular}{llllll}
\hline $\begin{array}{l}\text { model } \\
\text { type }\end{array}$ & $\begin{array}{l}\text { training } \\
\text { strategy }\end{array}$ & $\begin{array}{l}\text { featurization } \\
\text { strategy }\end{array}$ & $\begin{array}{l}\log P \\
\text { RMSE }\end{array}$ & $r^{2}$ & $\begin{array}{l}\text { unique } \\
\text { ID }\end{array}$ \\
\hline D-GIN & 0 & 8 & $0.510 \pm 0.026$ & $0.878 \pm 0.012$ & dg801 \\
D-GIN & 0 & 3 & $0.496 \pm 0.025$ & $0.885 \pm 0.012$ & dg302 \\
D-GIN & 0 & 6 & $0.493 \pm 0.024$ & $0.889 \pm 0.010$ & dg601 \\
D-GIN & 6 & 4 & $0.493 \pm 0.021$ & $0.886 \pm 0.011$ & dg461 \\
D-GIN & 6 & 7 & $0.541 \pm 0.028$ & $0.862 \pm 0.014$ & dg761 \\
D-GIN & 0 & 4 & $0.487 \pm 0.023$ & $0.891 \pm 0.012$ & dg401 \\
D-GIN & 6 & 8 & $0.477 \pm 0.025$ & $0.893 \pm 0.011$ & dg862 \\
D-GIN & 0 & 7 & $0.560 \pm 0.029$ & $0.852 \pm 0.015$ & dg701 \\
D-GIN & 0 & 5 & $0.663 \pm 0.034$ & $0.793 \pm 0.021$ & dg501 \\
D-GIN & 6 & 5 & $0.653 \pm 0.029$ & $0.802 \pm 0.018$ & dg561 \\
D-GIN & 6 & 3 & $0.472 \pm 0.020$ & $0.896 \pm 0.009$ & dg362 \\
D-GIN & 6 & 6 & $0.511 \pm 0.027$ & $0.878 \pm 0.013$ & dg662 \\
D-GIN cons. & 6 & 3 & $0.428 \pm 0.026$ & $0.914 \pm 0.010$ & dg362 cons. \\
D-GIN cons. & 6 & 5 & $0.502 \pm 0.027$ & $0.881 \pm 0.013$ & dg561 cons. \\
D-GIN cons. & 0 & 7 & $0.473 \pm 0.032$ & $0.894 \pm 0.013$ & dg701 cons. \\
D-GIN cons. & 0 & 5 & $0.515 \pm 0.033$ & $0.875 \pm 0.014$ & dg501 cons. \\
D-GIN cons. & 0 & 3 & $0.447 \pm 0.030$ & $0.906 \pm 0.012$ & dg302 cons. \\
D-GIN cons. & 6 & 8 & $0.433 \pm 0.030$ & $0.912 \pm 0.012$ & dg862 cons. \\
D-GIN cons. & 0 & 6 & $0.434 \pm 0.029$ & $0.911 \pm 0.011$ & dg601 cons. \\
D-GIN cons. & 0 & 4 & $0.439 \pm 0.026$ & $0.909 \pm 0.011$ & dg401 cons. \\
D-GIN cons. & 6 & 7 & $0.461 \pm 0.027$ & $0.900 \pm 0.012$ & dg761 cons. \\
D-GIN cons. & 0 & 8 & $0.452 \pm 0.030$ & $0.904 \pm 0.014$ & dg801 cons. \\
D-GIN cons. & 6 & 4 & $0.440 \pm 0.030$ & $0.909 \pm 0.012$ & dg461 cons. \\
D-GIN cons. & 6 & 6 & $0.442 \pm 0.026$ & $0.908 \pm 0.011$ & dg661 cons. \\
\hline
\end{tabular}


Table 23: Shows the $\log P$ RMSE and $r^{2}$ results for the D-MPNN model type used during this survey. The last column consist of the unique identify. Training strategy 0 represents a training strategy combining $\log \mathrm{D}, \log \mathrm{S}$ and $\log \mathrm{P}, 1$ represents a strategy combining $\log \mathrm{D}$ and $\log \mathrm{P}, 2$ stands for a combination of $\log \mathrm{D}$ and $\log \mathrm{S}, 3$ represents a strategy using $\log \mathrm{S}$ and $\log \mathrm{P}, 4$ represents a strategy using $\log \mathrm{D}, 5$ represents a strategy using $\log \mathrm{S}$ and 6 represents a strategy using $\log \mathrm{P}$.

\begin{tabular}{|c|c|c|c|c|c|}
\hline $\begin{array}{l}\text { model } \\
\text { type }\end{array}$ & $\begin{array}{l}\text { training } \\
\text { strategy }\end{array}$ & $\begin{array}{l}\text { featurization } \\
\text { strategy }\end{array}$ & $\begin{array}{l}\log P \\
\text { RMSE }\end{array}$ & $r^{2}$ & $\begin{array}{l}\text { unique } \\
\text { ID }\end{array}$ \\
\hline D-MPNN & 6 & 6 & $0.540 \pm 0.019$ & $0.863 \pm 0.012$ & dmp661 \\
\hline D-MPNN & 6 & 5 & $0.735 \pm 0.026$ & $0.746 \pm 0.019$ & dmp561 \\
\hline D-MPNN & 0 & 8 & $0.583 \pm 0.020$ & $0.845 \pm 0.012$ & dmp802 \\
\hline D-MPNN & 0 & 6 & $0.551 \pm 0.022$ & $0.861 \pm 0.012$ & dmp601 \\
\hline D-MPNN & 6 & 4 & $0.556 \pm 0.019$ & $0.856 \pm 0.011$ & dmp461 \\
\hline D-MPNN & 0 & 5 & $0.717 \pm 0.027$ & $0.758 \pm 0.021$ & dmp501 \\
\hline D-MPNN & 6 & 3 & $0.570 \pm 0.019$ & $0.847 \pm 0.012$ & dmp362 \\
\hline D-MPNN & 6 & 7 & $0.629 \pm 0.023$ & $0.815 \pm 0.014$ & dmp762 \\
\hline D-MPNN & 0 & 7 & $0.605 \pm 0.020$ & $0.828 \pm 0.012$ & dmp702 \\
\hline D-MPNN & 6 & 8 & $0.593 \pm 0.021$ & $0.834 \pm 0.014$ & dmp862 \\
\hline D-MPNN & 0 & 4 & $0.571 \pm 0.022$ & $0.851 \pm 0.012$ & dmp401 \\
\hline D-MPNN & 0 & 3 & $0.552 \pm 0.018$ & $0.857 \pm 0.011$ & dmp301 \\
\hline D-MPNN cons. & 6 & 5 & $0.533 \pm 0.031$ & $0.866 \pm 0.015$ & dmp562 cons. \\
\hline D-MPNN cons. & 0 & 6 & $0.444 \pm 0.024$ & $0.907 \pm 0.010$ & dmp601 cons. \\
\hline D-MPNN cons. & 6 & 6 & $0.452 \pm 0.026$ & $0.904 \pm 0.011$ & dmp661 cons. \\
\hline D-MPNN cons. & 0 & 5 & $0.524 \pm 0.031$ & $0.870 \pm 0.015$ & dmp501 cons. \\
\hline D-MPNN cons. & 0 & 3 & $0.461 \pm 0.027$ & $0.900 \pm 0.011$ & dmp301 cons. \\
\hline D-MPNN cons. & 0 & 4 & $0.463 \pm 0.027$ & $0.899 \pm 0.011$ & dmp401 cons. \\
\hline D-MPNN cons. & 6 & 3 & $0.464 \pm 0.026$ & $0.899 \pm 0.012$ & dmp362 cons. \\
\hline D-MPNN cons. & 6 & 8 & $0.471 \pm 0.028$ & $0.895 \pm 0.012$ & dmp862 cons. \\
\hline D-MPNN cons. & 0 & 8 & $0.475 \pm 0.027$ & $0.894 \pm 0.011$ & dmp802 cons. \\
\hline D-MPNN cons. & 0 & 7 & $0.484 \pm 0.028$ & $0.890 \pm 0.012$ & dmp701 cons. \\
\hline D-MPNN cons. & 6 & 4 & $0.454 \pm 0.026$ & $0.903 \pm 0.012$ & dmp462 cons. \\
\hline D-MPNN cons. & 6 & 7 & $0.488 \pm 0.027$ & $0.888 \pm 0.012$ & dmp761 cons. \\
\hline
\end{tabular}


Table 24: Shows the $\log$ P RMSE and $r^{2}$ results for the GIN model type used during this survey. The last column consist of the unique identify. Training strategy 0 represents a training strategy combining $\log \mathrm{D}, \log \mathrm{S}$ and $\log \mathrm{P}, 1$ represents a strategy combining $\log \mathrm{D}$ and $\log \mathrm{P}, 2$ stands for a combination of $\log \mathrm{D}$ and $\log \mathrm{S}, 3$ represents a strategy using $\log \mathrm{S}$ and $\log \mathrm{P}, 4$ represents a strategy using $\log \mathrm{D}, 5$ represents a strategy using $\log \mathrm{S}$ and 6 represents a strategy using $\log \mathrm{P}$.

\begin{tabular}{llllll}
\hline $\begin{array}{l}\text { model } \\
\text { type }\end{array}$ & $\begin{array}{l}\text { training } \\
\text { strategy }\end{array}$ & $\begin{array}{l}\text { featurization } \\
\text { strategy }\end{array}$ & $\begin{array}{l}\text { logP } \\
\text { RMSE }\end{array}$ & $r^{2}$ & unique \\
\hline GIN & 0 & 3 & $0.752 \pm 0.040$ & $0.742 \pm 0.025$ & g302 \\
GIN & 0 & 8 & $0.750 \pm 0.029$ & $0.738 \pm 0.018$ & g802 \\
GIN & 0 & 4 & $0.716 \pm 0.032$ & $0.758 \pm 0.019$ & g402 \\
GIN & 0 & 5 & $0.902 \pm 0.033$ & $0.624 \pm 0.026$ & g502 \\
GIN & 6 & 5 & $0.894 \pm 0.036$ & $0.626 \pm 0.026$ & g561 \\
GIN & 0 & 7 & $0.717 \pm 0.033$ & $0.758 \pm 0.019$ & g701 \\
GIN & 6 & 7 & $0.727 \pm 0.032$ & $0.753 \pm 0.022$ & g761 \\
GIN & 6 & 3 & $0.739 \pm 0.030$ & $0.744 \pm 0.019$ & g361 \\
GIN & 6 & 8 & $0.741 \pm 0.027$ & $0.743 \pm 0.018$ & g862 \\
GIN & 6 & 6 & $0.871 \pm 0.033$ & $0.643 \pm 0.024$ & g662 \\
GIN & 6 & 4 & $0.725 \pm 0.037$ & $0.753 \pm 0.025$ & g462 \\
GIN & 0 & 6 & $0.883 \pm 0.036$ & $0.640 \pm 0.026$ & g601 \\
GINcons. & 6 & 7 & $0.537 \pm 0.035$ & $0.864 \pm 0.016$ & g761 cons. \\
GINcons. & 6 & 8 & $0.548 \pm 0.034$ & $0.858 \pm 0.016$ & g861 cons. \\
GINcons. & 0 & 4 & $0.539 \pm 0.035$ & $0.863 \pm 0.015$ & g402 cons. \\
GINcons. & 0 & 7 & $0.539 \pm 0.034$ & $0.863 \pm 0.015$ & g701 cons. \\
GINcons. & 6 & 4 & $0.534 \pm 0.039$ & $0.865 \pm 0.018$ & g462 cons. \\
GINcons. & 0 & 6 & $0.612 \pm 0.038$ & $0.823 \pm 0.019$ & g601 cons. \\
GINcons. & 6 & 6 & $0.606 \pm 0.037$ & $0.827 \pm 0.019$ & g662 cons. \\
GINcons. & 6 & 3 & $0.544 \pm 0.035$ & $0.860 \pm 0.015$ & g362 cons. \\
GINcons. & 0 & 3 & $0.547 \pm 0.036$ & $0.859 \pm 0.015$ & g302 cons. \\
GINcons. & 0 & 8 & $0.555 \pm 0.033$ & $0.855 \pm 0.014$ & g802 cons. \\
GINcons. & 6 & 5 & $0.614 \pm 0.038$ & $0.822 \pm 0.019$ & g561 cons. \\
GINcons. & 0 & 5 & $0.618 \pm 0.037$ & $0.820 \pm 0.018$ & g502 cons. \\
\hline & & & & &
\end{tabular}


Table 25: Shows the logP RMSE and $r^{2}$ results for the non-GNN model types used during this survey. The last column consist of the unique identify. Training strategy 0 represents a training strategy combining $\log \mathrm{D}, \log \mathrm{S}$ and $\log \mathrm{P}, 1$ represents a strategy combining $\log \mathrm{D}$ and $\log \mathrm{P}, 2$ stands for a combination of $\log \mathrm{D}$ and $\log \mathrm{S}, 3$ represents a strategy using $\log \mathrm{S}$ and $\log \mathrm{P}, 4$ represents a strategy using $\log \mathrm{D}, 5$ represents a strategy using $\log \mathrm{S}$ and 6 represents a strategy using $\log \mathrm{P}$.

\begin{tabular}{llllll}
\hline $\begin{array}{l}\text { model } \\
\text { type }\end{array}$ & $\begin{array}{lllll}\text { training } \\
\text { strategy }\end{array}$ & $\begin{array}{l}\text { featurization } \\
\text { strategy }\end{array}$ & $\begin{array}{l}\log \mathrm{P} \\
\text { RMSE }\end{array}$ & $r^{2}$ & $\begin{array}{l}\text { unique } \\
\text { ID }\end{array}$ \\
\hline KNN & $\log \mathrm{P}$ & 13 & $0.939 \pm 0.029$ & $0.586 \pm 0.024$ & KNN13 \\
KNN & $\log \mathrm{P}$ & 14 & $0.995 \pm 0.029$ & $0.534 \pm 0.025$ & KNN14 \\
KNN & $\log \mathrm{P}$ & 15 & $1.065 \pm 0.032$ & $0.467 \pm 0.027$ & KNN15 \\
KNN & $\log \mathrm{P}$ & 10 & $1.082 \pm 0.035$ & $0.450 \pm 0.023$ & KNN10 \\
KNN & $\log \mathrm{P}$ & 12 & $1.087 \pm 0.033$ & $0.445 \pm 0.023$ & KNN12 \\
KNN & $\log \mathrm{P}$ & 16 & $1.100 \pm 0.034$ & $0.431 \pm 0.026$ & KNN16 \\
KNN & $\log \mathrm{P}$ & 11 & $1.103 \pm 0.035$ & $0.428 \pm 0.026$ & KNN11 \\
KNN & $\log \mathrm{P}$ & 17 & $0.744 \pm 0.019$ & $0.740 \pm 0.014$ & KNN17 \\
RF & $\log \mathrm{P}$ & 13 & $0.814 \pm 0.029$ & $0.688 \pm 0.023$ & RF13 \\
RF & $\log \mathrm{P}$ & 15 & $0.479 \pm 0.022$ & $0.892 \pm 0.010$ & RF15 \\
RF & $\log \mathrm{P}$ & 17 & $0.472 \pm 0.023$ & $0.895 \pm 0.010$ & RF17 \\
RF & $\log \mathrm{P}$ & 14 & $0.472 \pm 0.024$ & $0.895 \pm 0.011$ & RF14 \\
RF & $\log \mathrm{P}$ & 12 & $0.893 \pm 0.030$ & $0.625 \pm 0.021$ & RF12 \\
RF & $\log \mathrm{P}$ & 16 & $0.470 \pm 0.024$ & $0.896 \pm 0.011$ & RF16 \\
RF & $\log \mathrm{P}$ & 10 & $0.921 \pm 0.031$ & $0.601 \pm 0.022$ & RF10 \\
RF & $\log \mathrm{P}$ & 11 & $0.928 \pm 0.029$ & $0.595 \pm 0.022$ & RF11 \\
SVM & $\log \mathrm{P}$ & 14 & $0.572 \pm 0.021$ & $0.846 \pm 0.012$ & SVM14 \\
SVM & $\log \mathrm{P}$ & 12 & $0.809 \pm 0.027$ & $0.692 \pm 0.018$ & SVM12 \\
SVM & $\log \mathrm{P}$ & 16 & $0.628 \pm 0.020$ & $0.815 \pm 0.012$ & SVM16 \\
SVM & $\log \mathrm{P}$ & 17 & $0.493 \pm 0.030$ & $0.886 \pm 0.013$ & SVM17 \\
SVM & $\log \mathrm{P}$ & 10 & $0.833 \pm 0.029$ & $0.673 \pm 0.020$ & SVM10 \\
SVM & $\log \mathrm{P}$ & 11 & $0.827 \pm 0.028$ & $0.678 \pm 0.020$ & SVM11 \\
SVM & $\log \mathrm{P}$ & 13 & $0.782 \pm 0.029$ & $0.713 \pm 0.020$ & SVM13 \\
SVM & $\log \mathrm{P}$ & 15 & $0.602 \pm 0.021$ & $0.830 \pm 0.013$ & SVM15 \\
\hline
\end{tabular}

\section{References}

1. Yang, K.; Swanson, K.; Jin, W.; Coley, C.; Eiden, P.; Gao, H.; Guzman-Perez, A.; Hopper, T.; Kelley, B.; Mathea, M.; Palmer, A.; Settels, V.; Jaakkola, T.; Jensen, K.; Barzilay, R. Analyzing Learned Molecular Representations for Property Prediction. Journal of Chemical Information and Modeling 2019, 59, 3370-3388. doi:10.1021/acs.jcim.9b00237.

2. Hu, W.; Liu, B.; Gomes, J.; Zitnik, M.; Liang, P.; Pande, V.S.; Leskovec, J. Pre-training Graph Neural Networks. CoRR 2019, abs/1905.12265, [1905.12265].

3. Gilmer, J.; Schoenholz, S.S.; Riley, P.F.; Vinyals, O.; Dahl, G.E. Neural Message Passing for Quantum Chemistry 2017. [1704.01212]. doi:10.1002/nme.2457.

4. Wieder, O.; Kohlbacher, S.; Kuenemann, M.; Garon, A.; Ducrot, P.; Seidel, T.; Langer, T. A compact review of molecular property prediction with graph neural networks. Drug Discovery Today: Technologies 2020. doi:https://doi.org/10.1016/j.ddtec.2020.11.009.

5. Wu, Z.; Pan, S.; Chen, F.; Long, G.; Zhang, C.; Yu, P.S. A Comprehensive Survey on Graph Neural Networks 2019. [1901.00596].

6. Zhou, K.; Dong, Y.; Lee, W.S.; Hooi, B.; Xu, H.; Feng, J. Effective Training Strategies for Deep Graph Neural Networks 2020. pp. 1-26, [2006.07107].

7. Wu, Z.; Pan, S.; Chen, F.; Long, G.; Zhang, C.; Yu, P.S. A Comprehensive Survey on Graph Neural Networks. Technical report, [1901.00596v1].

8. Shang, C.; Liu, Q.; Chen, K.S.; Sun, J.; Lu, J.; Yi, J.; Bi, J. Edge Attention-based Multi-Relational Graph Convolutional Networks, 2018, [arXiv:stat.ML/1802.04944].

9. Liao, R.; Zhao, Z.; Urtasun, R.; Zemel, R.S. LanczosNet: Multi-scale deep graph convo-lutional networks. 7th International Conference on Learning Representations, ICLR 2019 2019, pp. 1-18, [arXiv:1901.01484v2].

10. Withnall, M.; Lindelöf, E.; Engkvist, O.; Chen, H. Building attention and edge message passing neural networks for bioactivity and physical-chemical property prediction. Journal of Cheminformatics 2020, 12. doi:10.1186/s13321-019-0407-y. 
11. Yuan, H.; Ji, S. StructPool: Structured Graph Pooling via Conditional Random Fields. International Conference on Learning Representations, 2020.

12. Hu, W. FOR P RE - TRAINING G RAPH N EURAL 2020. pp. 1-22, [arXiv:1905.12265v3].

13. Micheli, A. Neural network for graphs: A contextual constructive approach. IEEE Transactions on Neural Networks 2009, 20, 498-511. doi:10.1109/TNN.2008.2010350.

14. Lusci, A.; Pollastri, G.; Baldi, P. Deep architectures and deep learning in chemoinformatics: The prediction of aqueous solubility for drug-like molecules. Journal of Chemical Information and Modeling 2013, 53, 1563-1575. doi:10.1021/ci400187y.

15. Bruna, J.; Zaremba, W.; Szlam, A.; LeCun, Y. Spectral Networks and Locally Connected Networks on Graphs, 2014, [arXiv:cs.LG/1312.6203].

16. Duvenaud, D.; Maclaurin, D.; Aguilera-Iparraguirre, J.; Gómez-Bombarelli, R.; Hirzel, T.; Aspuru-Guzik, A.; Adams, R.P. Convolutional networks on graphs for learning molecular fingerprints. Advances in Neural Information Processing Systems 2015, 2015-Janua, 2224-2232, [1509.09292].

17. Coley, C.W.; Barzilay, R.; Green, W.H.; Jaakkola, T.S.; Jensen, K.F. Convolutional Embedding of Attributed Molecular Graphs for Physical Property Prediction. Journal of Chemical Information and Modeling 2017, 57, 1757-1772. doi:10.1021/acs.jcim.6b00601.

18. Wu, Z.; Ramsundar, B.; Feinberg, E.N.; Gomes, J.; Geniesse, C.; Pappu, A.S.; Leswing, K.; Pande, V. MoleculeNet : A Benchmark for Molecular Machine Learning arXiv : 1703 . 00564v3 [ cs . LG ] 26 Oct 2018. [arXiv:1703.00564v3].

19. Bouritsas, G.; Frasca, F.; Zafeiriou, S.; Bronstein, M.M. Improving Graph Neural Network Expressivity via Subgraph Isomorphism Counting 2020. [arXiv:cs.LG/2006.09252].

20. Xu, K.; Jegelka, S.; Hu, W.; Leskovec, J. How powerful are graph neural networks? 7th International Conference on Learning Representations, ICLR 2019 2019, pp. 1-17, [arXiv:1810.00826v3].

21. Weisfeiler, B.Y.; Leman, A.A. A reduction of a graph to a canonical form and an algebra arising during this reduction. NauchnoTechnicheskaya Informatsia , 2(9) 1968, pp. 2-16.

22. Morris, C.; Ritzert, M.; Fey, M.; Hamilton, W.L.; Lenssen, J.E.; Rattan, G.; Grohe, M. Weisfeiler and Leman Go Neural: HigherOrder Graph Neural Networks. Proceedings of the AAAI Conference on Artificial Intelligence 2019, 33, 4602-4609, [1810.02244]. doi:10.1609/aaai.v33i01.33014602.

23. Dwivedi, V.P.; Joshi, C.K.; Laurent, T.; Bengio, Y.; Bresson, X. Benchmarking Graph Neural Networks 2020. [2003.00982].

24. Mayr, A.; Klambauer, G.; Unterthiner, T.; Steijaert, M.; Wegner, J.K.; Ceulemans, H.; Clevert, D.A.; Hochreiter, S. Largescale comparison of machine learning methods for drug target prediction on ChEMBL. Chemical Science 2018, 9, 5441-5451. doi:10.1039/c8sc00148k.

25. Errica, F.; Podda, M.; Bacciu, D.; Micheli, A. A Fair Comparison of Graph Neural Networks for Graph Classification. CoRR 2019, abs/1912.09893, [1912.09893].

26. Shchur, O.; Mumme, M.; Bojchevski, A.; Günnemann, S. Pitfalls of Graph Neural Network Evaluation. CoRR 2018, abs/1811.05868, [1811.05868].

27. Neal, B. On the Bias-Variance Tradeoff: Textbooks Need an Update, 2019, [arXiv:cs.LG/1912.08286].

28. Cui, Q.; Lu, S.; Ni, B.; Zeng, X.; Tan, Y.; Chen, Y.D.; Zhao, H. Improved Prediction of Aqueous Solubility of Novel Compounds by Going Deeper With Deep Learning. Frontiers in Oncology 2020, 10, 121. doi:10.3389/fonc.2020.00121.

29. Graph Networks. https://github.com/spudlig/graph_networks. Accessed: 2021-08-01.

30. RDKit. https://www.rdkit.org/. Accessed: 2020-09-01.

31. CDPKit. https://github.com/aglanger/CDPKit. Accessed: 2020-01-30.

32. Abadi, M.; Agarwal, A.; Barham, P.; Brevdo, E.; Chen, Z.; Citro, C.; Corrado, G.S.; Davis, A.; Dean, J.; Devin, M.; Ghemawat, S.; Goodfellow, I.; Harp, A.; Irving, G.; Isard, M.; Jia, Y.; Jozefowicz, R.; Kaiser, L.; Kudlur, M.; Levenberg, J.; Mané, D.; Monga, R.; Moore, S.; Murray, D.; Olah, C.; Schuster, M.; Shlens, J.; Steiner, B.; Sutskever, I.; Talwar, K.; Tucker, P.; Vanhoucke, V.; Vasudevan, V.; Viégas, F.; Vinyals, O.; Warden, P.; Wattenberg, M.; Wicke, M.; Yu, Y.; Zheng, X. TensorFlow: Large-Scale Machine Learning on Heterogeneous Systems, 2015. Software available from tensorflow.org.

33. Pedregosa, F.; Varoquaux, G.; Gramfort, A.; Michel, V.; Thirion, B.; Grisel, O.; Blondel, M.; Prettenhofer, P.; Weiss, R.; Dubourg, V.; Vanderplas, J.; Passos, A.; Cournapeau, D.; Brucher, M.; Perrot, M.; Duchesnay, E. Scikit-learn: Machine Learning in Python. Journal of Machine Learning Research 2011, 12, 2825-2830.

34. Xie, Y.; Gong, M.; Gao, Y.; Qin, A.K.; Fan, X. A Multi-Task Representation Learning Architecture for Enhanced Graph Classification. Frontiers in Neuroscience 2020, 13, 1395. doi:10.3389/fnins.2019.01395.

35. Seltzer, M.L.; Droppo, J. Multi-task learning in deep neural networks for improved phoneme recognition. 2013 IEEE International Conference on Acoustics, Speech and Signal Processing, 2013, pp. 6965-6969. doi:10.1109/ICASSP.2013.6639012.

36. Hashimoto, K.; Xiong, C.; Tsuruoka, Y.; Socher, R. A Joint Many-Task Model: Growing a Neural Network for Multiple NLP Tasks. CoRR 2016, abs/1611.01587, [1611.01587]. 